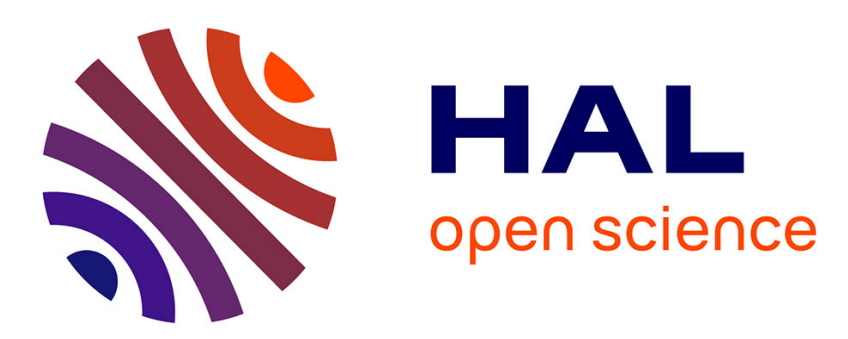

\title{
Topological sensitivity for 3D elastodynamic and acoustic inverse scattering in the time domain \\ Marc Bonnet
}

\section{To cite this version:}

Marc Bonnet. Topological sensitivity for 3D elastodynamic and acoustic inverse scattering in the time domain. Computer Methods in Applied Mechanics and Engineering, 2006, 195, pp.5239-5254. 10.1016/j.cma.2005.10.026 . hal-00092360

\section{HAL Id: hal-00092360 https://hal.science/hal-00092360}

Submitted on 9 Aug 2008

HAL is a multi-disciplinary open access archive for the deposit and dissemination of scientific research documents, whether they are published or not. The documents may come from teaching and research institutions in France or abroad, or from public or private research centers.
L'archive ouverte pluridisciplinaire HAL, est destinée au dépôt et à la diffusion de documents scientifiques de niveau recherche, publiés ou non, émanant des établissements d'enseignement et de recherche français ou étrangers, des laboratoires publics ou privés. 


\title{
Topological sensitivity for $3 \mathrm{D}$ elastodynamic and acoustic inverse scattering in the time domain. *
}

\author{
Marc Bonnet \\ Solid Mechanics Laboratory (CNRS UMR 7649), Department of Mechanics \\ Ecole Polytechnique, 91128 Palaiseau cedex, France
}

\begin{abstract}
Building on previous work for 3D inverse scattering in the frequency domain, this article develops the concept of topological derivative for 3D elastic and acousticwave imaging of media of arbitrary geometry using data in the time domain. The topological derivative, which quantifies the sensitivity of the cost functional associated with the inverse scattering problem due to the creation at a specified location of an infinitesimal hole (for the elastodynamic case) or rigid inclusion (for the acoustic case), is found to be expressed in terms of the time convolution of the free field and a supplementary adjoint field. The derivation of the topological derivative follows the generic pattern proposed in previous studies, which is transposable to a variety of other physical problems. A numerical example, where the featured cost function is defined in terms of synthetic data arising from the scattering of plane acoustic waves by a rigid spherical inclusion, illustrates the utility of the topological derivative concept for defect identification using time-varying data.
\end{abstract}

Key words: Topological derivative, Inverse scattering, Convolution, Linear acoustics, linear elastodynamics, Adjoint field method

\section{Introduction}

Three-dimensional imaging of objects hidden in a solid using elastic waves is a topic of interest in a number of applications ranging from nondestructive material testing to underground object detection. Usually, such imaging is performed by minimizing a cost function measuring the discrepancy between

ऋ Computer Methods in Applied Mechanics and Engineering 195:5239-5254 (2006) Email address: bonnet@lms.polytechnique.fr (Marc Bonnet). 
the experimental data and its computed counterpart for an assumed configuration of the hidden scatterer (or system thereof). Numerical minimization techniques then require successive evaluations of the cost function for incrementally updated such configurations, until convergence is reached. Each cost function evaluation entails solving the forward elastodynamic problem for a given scatterer. In the context of three-dimensional imaging, these approaches bear substantial computational costs associated with the elastodynamic forward solver. Computing time considerations in particular make optimization methods based on global search currently impractical for three-dimensional elastodynamic inverse scattering problems. More traditional gradient-based optimization algorithms have been shown to be a computationally reasonable alternative for this class of inverse problems, especially when enhanced by analytical shape sensitivity estimates $[1,2]$. However, performance of such blind minimization techniques is dependent on choosing adequately the initial guess (location, topology and geometry) of the hidden object(s), because of the highly non-convex character of the cost function. As a result, their stand-alone use may not be sufficient for robust 3D elastodynamic imaging.

These considerations have prompted the definition of preliminary probing techniques, which aim at delineating in a computationally faster way the hidden object(s). Such techniques have been defined on the basis of either the linear sampling [3,4], an avenue not pursued here, or the concept of topological derivative, whereby the sensitivity of a cost function with respect to the creation of an infinitesimal object of small characteristic radius $\varepsilon$ at a prescribed location $\boldsymbol{x}^{\circ}$ inside the reference, i.e. cavity-free counterpart of the probed body, is quantified as a function of $\boldsymbol{x}^{\circ}$. The concept of topological derivative first appeared in [5] and [6] in the context of topological optimization of mechanical structures (see [7] for a description of that subject), allowing to define algorithms where "excess" material is iteratively removed until a satisfactory shape and topology is reached [8]. Later, its rigorous mathematical formulation has been established within the framework of elastostatic problems and Laplace equation $[8,9]$. However, beyond applications to the topology and shape optimization of structures which were its initial motivation, the topological derivative is also expected to facilitate the minimization-based solution of inverse scattering problems by providing, through a first-order approximation, a rational basis for establishing initial "guesses" for hidden defects (in terms of their number, location, and geometry). This idea has been considered in the context of frequency-domain 3D elastodynamic scattering in semi-infinite and infinite domains [10] and in bounded bodies [11]. Related studies include [12,13] for 2D elastostatics and [14] for 2D linear acoustics.

This article, which builds on earlier works $[10,11]$, extends the concept of topological derivative and its application to cavity identification to the case of 3D time-domain elastodynamic and acoustic scattering in domains of arbitrary shape. The topological derivative $\mathcal{T}\left(\boldsymbol{x}^{\circ}\right)$ is hence established for objective 
functions having the form of an integral over the body's external boundary and the time interval of interest, and whose density involves the solution of the forward scattering problem. This format constitutes a natural generalization of the least-squares misfit function integrated in time, often used in identification problems. The topological derivative is then established with the help of a boundary integral equation (BIE) formulation of the scattering problem, which facilitates the analysis of the limiting case of a cavity of vanishing size. It is found to be given by a formula involving the time convolution of the free field (the response of the reference body to the probing load) and the adjoint field (the response of the reference body to the adjoint load, which is defined in terms of the cost function density). A similar, simpler, formula is obtained for the nucleation of infinitesimal rigid inclusions in 3D linear acoustic media.

The present study emphasizes the development of topological sensitivity as a preparatory tool for more accurate, gradient-based solution algorithms. The practical computation of the field $\mathcal{T}\left(\boldsymbol{x}^{\circ}\right)$ may be performed by any of the usual numerical solution techniques, such as the finite element method (FEM), the finite difference method (FDM) or the boundary element method (BEM). In such framework, the computational cost entailed by the topological sensitivity calculation is of the order of one forward elastodynamic solution, and therefore modest compared to that of the subsequent minimization of a cost function. Another possibility, not examined here, consists of defining an imaging algorithm entirely based on the topological derivative, in which matter removal is performed iteratively and $\mathcal{T}$ is updated after each matter removal step.

This article is organized as follows. Formulations and notation for the forward and inverse elastodynamic problems of interest are reviewed in Section 2. The concept of topological derivative for the present context is defined in Section 3. A formula for the topological derivative expressed in terms of the free and adjoint fields is established in Section 4 in the context of 3D elastodynamic scattering. Then, in Section 5, a similar, simpler, formula is obtained for 3D acoustic scattering. Additional issues are discussed in Section 6. A numerical example demonstrating the utility of topological derivative, where an unknown rigid spherical obstacle is sought from the acoustic pressure recorded at remote sensors as a function of time, is presented in Section 7 .

\section{$2 \quad$ Forward and inverse problems}

Let $\Omega$ denote a finite elastic solid (hereinafter referred to as the reference solid) bounded by the external surface $S$ and characterized by the shear modulus $\mu$, Poisson's ratio $\nu$ and mass density $\rho$. Moreover, let $\Omega_{\Gamma}$ denote a generic cavitated solid having the same external boundary $S$ as $\Omega$ and containing a cavity system occupying the (possibly multiply-connected) region $B$ with 
boundary $\Gamma$, so that $\Omega_{\Gamma}=\Omega \backslash \bar{B}$, where $\bar{B}$ denotes the closure of $B$. On assuming that (i) the cavity is stress-free, (ii) the cavitated solid is at rest at initial time $t=0$, and (iii) a traction distribution $\boldsymbol{p}(\boldsymbol{\xi}, t)$ is applied on a part $S_{\mathrm{N}}$ of the external surface $S$, an elastodynamic state $\boldsymbol{u}_{\Gamma}(\boldsymbol{\xi}, t)$ arises which satisfies the following set of field equations, boundary and initial conditions, collectively referred to as $\mathcal{P}(\Gamma)$ :

$$
\mathcal{P}(\Gamma):\left\{\begin{aligned}
{\left[\mathcal{L}(\mathcal{C}, \rho) \boldsymbol{u}_{\Gamma}\right](\boldsymbol{\xi}, t) } & =\mathbf{0} & & (\boldsymbol{\xi} \in \Omega, t \geq 0), \\
\boldsymbol{t}\left[\boldsymbol{u}_{\Gamma}\right](\boldsymbol{\xi}, t) & =\boldsymbol{p}(\boldsymbol{\xi}, t) & & \left(\boldsymbol{\xi} \in S_{\mathrm{N}}, t \geq 0\right), \\
\boldsymbol{t}\left[\boldsymbol{u}_{\Gamma}\right](\boldsymbol{\xi}, t) & =\mathbf{0} & & (\boldsymbol{\xi} \in \Gamma, t \geq 0), \\
\boldsymbol{u}_{\Gamma}(\boldsymbol{\xi}, t) & =\mathbf{0} & & \left(\boldsymbol{\xi} \in S_{\mathrm{D}}, t \geq 0\right), \\
\boldsymbol{u}_{\Gamma}(\boldsymbol{\xi}, 0)=\dot{\boldsymbol{u}}_{\Gamma}(\boldsymbol{\xi}, 0) & =\mathbf{0} & & (\boldsymbol{\xi} \in \Omega)
\end{aligned}\right.
$$

where $\boldsymbol{\xi}$ denotes the position vector and $\mathcal{L}(\mathcal{C}, \rho)$ is the governing partial differential operator of linear elastodynamics, i.e.

$$
[\mathcal{L}(\mathcal{C}, \rho) \boldsymbol{w}](\boldsymbol{\xi}, t)=\nabla \cdot(\mathcal{C}: \nabla \boldsymbol{w})(\boldsymbol{\xi}, t)-\rho \ddot{\boldsymbol{w}}(\boldsymbol{\xi}, t)
$$

with () indicating a partial derivative w.r.t. time. Here, the external surface $S$ is divided into complementary subsets $S_{\mathrm{N}}$ and $S_{\mathrm{D}}$ supporting prescribed tractions and displacements, respectively; $\boldsymbol{t}[\boldsymbol{w}] \equiv \boldsymbol{\sigma}[\boldsymbol{w}] \cdot \boldsymbol{n}=(\mathcal{C}: \nabla \boldsymbol{w}) \cdot \boldsymbol{n}$ denotes the traction vector associated with a generic displacement $\boldsymbol{w}$ through Hooke's law, and

$$
\mathcal{C}=2 \mu\left[\frac{1+\nu}{1-2 \nu} \mathcal{J}+\mathcal{K}\right]
$$

is the isotropic elasticity tensor, where the fourth-order tensors $\mathcal{J}$ and $\mathcal{K}$ are defined through

$$
3 \mathcal{J}=\boldsymbol{I}_{2} \otimes \boldsymbol{I}_{2}, \quad \mathcal{K}=\mathcal{I}-\mathcal{J}
$$

with $\boldsymbol{I}_{2}$ and $\boldsymbol{I}$ symbolizing the second-order and symmetric fourth-order identity tensors, respectively. Recall that $\mathcal{J}$ and $\mathcal{K}$ are associated with the decomposition of a second-order symmetric tensor into its spherical and deviatoric parts, and have the well-known orthonormality properties

$$
\mathcal{J}: \mathcal{J}=\mathcal{J}, \quad \mathcal{K}: \mathcal{K}=\mathcal{K}, \quad \mathcal{J}: \mathcal{K}=\mathbf{0}
$$

Let us consider objective functions $J\left(\Omega_{\Gamma}\right)$ having the generic format

$$
J\left(\Omega_{\Gamma}\right)=\int_{0}^{T} \int_{S^{\text {obs }}} \varphi\left(\boldsymbol{u}_{\Gamma}(\boldsymbol{\xi}, t), \boldsymbol{\xi}, t\right) \mathrm{d} \Gamma_{\xi} \mathrm{d} t
$$

where $S^{\text {obs }} \subseteq S$ (i.e. the support of the integral is a part of the external boundary), and the function $\varphi(\boldsymbol{w}, \boldsymbol{\xi}, t)$ is differentiable w.r.t. its first argument. Such objective functions, and their extremalization with respect to the cavity system symbolized by $\Gamma$, may arise in connection with e.g. the optimization of structures with respect to their shape [15] or topology [7], or with 
inverse problems in defect identification. In this article, cavity identification inverse problems are considered for the sake of definiteness, but the main results will in fact pertain to any objective function of type (6) independently of its practical motivation.

The identification of embedded objects (here, cavities) is usually based on the availability of overdetermined data on the external boundary. Here, measured values $\boldsymbol{u}^{\text {obs }}(\boldsymbol{\xi}, t)$ of displacements are assumed to be available over the measurement surface $S^{\text {obs }} \subseteq S_{\mathrm{N}}$. Ideally (i.e. assuming that the physics is exactly described by the chosen linear elastodynamics setting and that no measurement errors are present), the observations $\boldsymbol{u}^{\text {obs }}(\boldsymbol{\xi}, t)$ are the trace on $S^{\text {obs }} \times[0, T]$ of the elastodynamic displacement $\boldsymbol{u}^{\text {true }}$ which solves problem $\mathcal{P}\left(\Gamma^{\text {true }}\right)$, where $\Gamma^{\text {true }}$ symbolizes the (unknown) cavity system to be identified. For such inverse problems, least-squares misfit functions of the form

$$
J_{\mathrm{LS}}\left(\Omega_{\Gamma}\right)=\int_{0}^{T} \int_{S^{\mathrm{obs}}}\left|\boldsymbol{u}_{\Gamma}(\boldsymbol{\xi}, t)-\boldsymbol{u}^{\mathrm{obs}}(\boldsymbol{\xi}, t)\right|^{2} \mathrm{~d} \Gamma_{\xi} \mathrm{d} t
$$

i.e.

$$
\varphi(\boldsymbol{w}, \boldsymbol{\xi}, t)=\left|\boldsymbol{w}-\boldsymbol{u}^{\mathrm{obs}}(\boldsymbol{\xi}, t)\right|^{2}
$$

are typically considered. More elaborate misfit functions, which usually adhere to the format (6), may involve norms other than the $L^{2}$-norm or stem from approaches based on e.g. Bayesian inference [16] or entropy maximization; such variations will not be investigated in this article.

A vast array of algorithmic approaches is available for performing the minimization (or, more generally, extremalization) of cost functions (6) or (7). Such methods are iterative and require a number of evaluations of $J\left(\Omega_{\Gamma}\right)$. Traditional gradient-based minimization techniques, such as the conjugate gradient or the quasi-Newton methods, may converge after only a moderate number of cost functions evaluations (if $\Gamma$ can be described in terms of a few geometrical parameters) but reach a local minimum which often depends on how the necessary initial guess for $\Gamma$ has been chosen. More recently, global search techniques, e.g. evolutionary algorithms [17] or sampling methods based on the Metropolis algorithm [16] have been proposed. They perform a global search (i.e. identify absolute and/or multiple minima), but at the cost of very large numbers of cost functions evaluations.

\section{Topological derivative}

The topological derivative is a mathematical tool aimed at quantifying the sensitivity of a functional of type (6) to the appearance of a very small cavity centered at a specified location $\boldsymbol{x}^{\circ}$ in the reference body $\Omega$. Moreover, this sensitivity is to be sought as a function of the infinitesimal cavity location $\boldsymbol{x}^{\circ}$. 
To this end, let $B_{\varepsilon}\left(\boldsymbol{x}^{\circ}\right)=\boldsymbol{x}^{\circ}+\varepsilon \mathscr{B}$, where $\mathscr{B} \subset \mathbb{R}^{3}$ is a fixed bounded open set with boundary $\mathscr{S}$ and volume $|\mathscr{B}|$ containing the origin, define the region of space occupied by a cavity of (small) size $0<\varepsilon \ll \operatorname{Diam}(\Omega)$ containing a specified sampling point $\boldsymbol{x}^{\circ}$. Following $[8,9]$, one seeks the asymptotic behavior of $J\left(\Omega_{\varepsilon}\right)$ for infinitesimal $\varepsilon>0$, where $\Omega_{\varepsilon}=\Omega_{\Gamma_{\varepsilon}}=\Omega \backslash \overline{B_{\varepsilon}}\left(\boldsymbol{x}^{\circ}\right), \Gamma_{\varepsilon}$ is the boundary of $B_{\varepsilon}$ and $\overline{B_{\varepsilon}}\left(\boldsymbol{x}^{\circ}\right)$ is the closure of $B_{\varepsilon}\left(\boldsymbol{x}^{\circ}\right)$. The topological derivative $\mathcal{T}\left(\boldsymbol{x}^{\circ}\right)$ of the cost functional $J(\Omega)$ at $\boldsymbol{x}^{\circ}$ for a cavity-free body is hence defined through the expansion:

$$
\begin{aligned}
& J\left(\Omega_{\varepsilon}\right)=J(\Omega)+\eta(\varepsilon)|\mathscr{B}| \mathcal{T}\left(\boldsymbol{x}^{\circ}\right)+o(\eta(\varepsilon)) \\
&\left(\varepsilon \ll \operatorname{Diameter}(\Omega), \quad B_{\varepsilon}\left(\boldsymbol{x}^{\circ}\right) \subset \Omega\right)
\end{aligned}
$$

where the function $\eta(\varepsilon)$, which characterizes the asymptotic behaviour of $J\left(\Omega_{\varepsilon}\right)-J(\Omega)$, is expected to vanish in the limit $\varepsilon \rightarrow 0$ and is to be determined. One may note that this definition is not restricted to spherical infinitesimal cavities (for which $\mathscr{B}$ is the unit ball, $\mathscr{S}$ the unit sphere and $|\mathscr{B}|=4 \pi / 3$ ). The value $\mathcal{T}\left(\boldsymbol{x}^{\circ}\right)$ is then expected to depend on the shape of $\mathscr{B}$.

The evaluation of $J\left(\Omega_{\varepsilon}\right)$ requires the elastodynamic solution $\boldsymbol{u}^{\varepsilon}$ to the forward problem $\mathcal{P}\left(\Gamma_{\varepsilon}\right)$ defined by (1). To this end, it is convenient and customary to decompose $\boldsymbol{u}^{\varepsilon}$ according to

$$
\boldsymbol{u}^{\varepsilon}(\boldsymbol{\xi}, t)=\boldsymbol{u}(\boldsymbol{\xi}, t)+\tilde{\boldsymbol{u}}^{\varepsilon}(\boldsymbol{\xi}, t)
$$

where $\boldsymbol{u}$ is the free field defined as the response of the void-free (reference) solid $\Omega$ to the prescribed excitation (here, the traction $\boldsymbol{p}$ applied over $S_{\mathrm{N}}$ ), so that

$$
\begin{aligned}
{[\mathcal{L}(\mathcal{C}, \rho) \boldsymbol{u}](\boldsymbol{\xi}, t) } & =\mathbf{0} & & (\boldsymbol{\xi} \in \Omega, t \geq 0), \\
\boldsymbol{t}[\boldsymbol{u}](\boldsymbol{\xi}, t) & =\boldsymbol{p}(\boldsymbol{\xi}, t) & & \left(\boldsymbol{\xi} \in S_{\mathrm{N}}, t \geq 0\right), \\
\boldsymbol{u}(\boldsymbol{\xi}, t) & =\mathbf{0} & & \left(\boldsymbol{\xi} \in S_{\mathrm{D}}, t \geq 0\right), \\
\boldsymbol{u}(\boldsymbol{\xi}, 0)=\boldsymbol{\boldsymbol { u }}(\boldsymbol{\xi}, 0) & =\mathbf{0} & & (\boldsymbol{\xi} \in \Omega)
\end{aligned}
$$

and $\tilde{\boldsymbol{u}}^{\varepsilon}$ denotes the scattered field, which solves the boundary-initial value problem

$$
\begin{aligned}
{\left[\mathcal{L}(\mathcal{C}, \rho) \tilde{\boldsymbol{u}}^{\varepsilon}\right](\boldsymbol{\xi}, t) } & =\mathbf{0} & & \left(\boldsymbol{\xi} \in \Omega_{\varepsilon}, t \geq 0\right), \\
\boldsymbol{t}\left[\tilde{\boldsymbol{u}}^{\varepsilon}\right](\boldsymbol{\xi}, t) & =-\boldsymbol{t}[\boldsymbol{u}](\boldsymbol{\xi}, t) & & \left(\boldsymbol{\xi} \in \Gamma_{\varepsilon}, t \geq 0\right), \\
\boldsymbol{t}\left[\tilde{\boldsymbol{u}}^{\varepsilon}\right](\boldsymbol{\xi}, t) & =\mathbf{0} & & \left(\boldsymbol{\xi} \in S_{\mathrm{N}}, t \geq 0\right), \\
\tilde{\boldsymbol{u}}^{\varepsilon}(\boldsymbol{\xi}, t) & =\mathbf{0} & & \left(\boldsymbol{\xi} \in S_{\mathrm{D}}, t \geq 0\right), \\
\tilde{\boldsymbol{u}}^{\varepsilon}(\boldsymbol{\xi}, 0)=\dot{\tilde{\boldsymbol{u}}}^{\varepsilon}(\boldsymbol{\xi}, 0) & =\mathbf{0} & & (\boldsymbol{\xi} \in \Omega)
\end{aligned}
$$

The scattered field is expected to vanish in the limit $\varepsilon \rightarrow 0$, i.e.

$$
\lim _{\varepsilon \rightarrow 0}\left|\tilde{\boldsymbol{u}}^{\varepsilon}(\boldsymbol{x}, t)\right|=0 \quad\left(\boldsymbol{x} \in \Omega_{\Gamma}, \boldsymbol{x} \neq \boldsymbol{x}^{\circ} ; \quad t \in[0, T]\right)
$$

whereas the free-field, by its definition (10), does not depend on $\varepsilon$. 
On the basis of (12), one may expand the objective function density as

$$
\varphi\left(\boldsymbol{u}^{\varepsilon}(\boldsymbol{\xi}, t), \boldsymbol{\xi}, t\right)=\varphi(\boldsymbol{u}(\boldsymbol{\xi}, t), \boldsymbol{\xi}, t)+\frac{\partial \varphi}{\partial \boldsymbol{u}}(\boldsymbol{u}(\boldsymbol{\xi}, t), \boldsymbol{\xi}, t) \cdot \tilde{\boldsymbol{u}}^{\varepsilon}(\boldsymbol{\xi}, t)+o\left(\left|\tilde{\boldsymbol{u}}^{\varepsilon}(\boldsymbol{\xi}, t)\right|\right)
$$

so that the perturbation of the objective function caused by the nucleation of an infinitesimal cavity of characteristic size $\varepsilon$ located at $\boldsymbol{x}^{\circ}$ is expressed as

$$
J\left(\Omega_{\varepsilon}\right)-J(\Omega)=\int_{0}^{T} \int_{S^{\mathrm{obs}}} \frac{\partial \varphi}{\partial \boldsymbol{u}}(\boldsymbol{u}(\boldsymbol{\xi}, t), \boldsymbol{\xi}, t) \cdot \tilde{\boldsymbol{u}}^{\varepsilon}(\boldsymbol{\xi}, t) \mathrm{d} \Gamma_{\xi} \mathrm{d} t+o\left(\left|\tilde{\boldsymbol{u}}^{\varepsilon}\right|\right)
$$

Definition (8) therefore requires that the topological derivative of $J(\Omega)$ and the asymptotic behaviour $\eta(\varepsilon)$ be identified on the basis of

$$
\int_{0}^{T} \int_{S^{\mathrm{obs}}} \frac{\partial \varphi}{\partial \boldsymbol{u}}(\boldsymbol{u}(\boldsymbol{\xi}, t), \boldsymbol{\xi}, t) \cdot \tilde{\boldsymbol{u}}^{\varepsilon}(\boldsymbol{\xi}, t) \mathrm{d} \Gamma_{\xi} \mathrm{d} t=\mathcal{T}\left(\boldsymbol{x}^{\circ}\right) \eta(\varepsilon)+o(\eta(\varepsilon)) .
$$

\section{Adjoint field approach}

As in more traditional parameter or shape sensitivity analysis, the purpose of the adjoint field approach is to evaluate the topological sensitivity, i.e. the limiting process defined by (14), without having to actually compute $\tilde{\boldsymbol{u}}^{\varepsilon}(\boldsymbol{\xi}, t)$, the sensitivity of the primary field variable. For that purpose, it is useful to interpret the integral in (14) in terms of a reciprocity identity.

\subsection{Elastodynamic reciprocity identity}

In the present context of linear elastodynamics in the time domain, it is well known $[18,19]$ that any pair of elastodynamic displacement fields $\boldsymbol{w}_{a}(\boldsymbol{\xi}, t)$, $\boldsymbol{w}_{b}(\boldsymbol{\xi}, t)$ defined over a regular domain $V$ with boundary $\partial V$ and satisfying the homogeneous field equations

$$
\left[\mathcal{L}(\mathcal{C}, \rho) \boldsymbol{w}_{a}\right](\boldsymbol{\xi}, t)=\mathbf{0}, \quad\left[\mathcal{L}(\mathcal{C}, \rho) \boldsymbol{w}_{b}\right](\boldsymbol{\xi}, t)=\mathbf{0}, \quad(\boldsymbol{\xi} \in V, \quad t \geq 0)
$$

and initial conditions

$$
\boldsymbol{w}_{a}(\boldsymbol{\xi}, 0)=\dot{\boldsymbol{w}}_{a}(\boldsymbol{\xi}, 0)=\mathbf{0}, \quad \boldsymbol{w}_{b}(\boldsymbol{\xi}, 0)=\dot{\boldsymbol{w}}_{b}(\boldsymbol{\xi}, 0)=\mathbf{0}, \quad(\boldsymbol{\xi} \in V)
$$

verifies the dynamical reciprocity identity

$$
\int_{\partial V}\left\{\boldsymbol{t}\left[\boldsymbol{w}_{a}\right] \star \boldsymbol{w}_{b}-\boldsymbol{t}\left[\boldsymbol{w}_{b}\right] \star \boldsymbol{w}_{a}\right\}(\boldsymbol{\xi}, t) \mathrm{d} \Gamma_{\xi}=0 \quad(t \geq 0)
$$

where $\star$ denote the time convolution, defined for any time instant $t \geq 0$ by:

$$
[\boldsymbol{a} \star \boldsymbol{b}](\boldsymbol{\xi}, t)=\int_{0}^{t} \boldsymbol{a}(\boldsymbol{\xi}, \tau) \cdot \boldsymbol{b}(\boldsymbol{\xi}, t-\tau) \mathrm{d} \tau=\int_{0}^{t} \boldsymbol{a}(\boldsymbol{\xi}, t-\tau) \cdot \boldsymbol{b}(\boldsymbol{\xi}, \tau) \mathrm{d} \tau
$$


where the inner product appearing in the integral is defined according to the order of the tensor fields $\boldsymbol{a}$ and $\boldsymbol{b}$ so that $\boldsymbol{a} \cdot \boldsymbol{b}$ is a scalar. The following property of the time convolution is recalled for later reference:

$$
[\ddot{\boldsymbol{a}} \star \boldsymbol{b}](\boldsymbol{\xi}, t)=[\dot{\boldsymbol{a}} \star \dot{\boldsymbol{b}}](\boldsymbol{\xi}, t)=[\boldsymbol{a} \star \ddot{\boldsymbol{b}}](\boldsymbol{\xi}, t)
$$

where the dot symbol (') indicates a partial derivative w.r.t. the time argument.

\subsection{Objective function perturbation expressed in terms of adjoint field}

Now, getting back to the topic of topological sensitivity analysis, define the adjoint field $\hat{\boldsymbol{u}}$ as the solution of the elastodynamic boundary-initial value problem

$$
\begin{aligned}
{[\mathcal{L}(\mathcal{C}, \rho) \hat{\boldsymbol{u}}](\boldsymbol{\xi}, t) } & =\mathbf{0} & & (\boldsymbol{\xi} \in \Omega, 0 \leq t \leq T), \\
\boldsymbol{t}[\hat{\boldsymbol{u}}](\boldsymbol{\xi}, t) & =\frac{\partial \varphi}{\partial \boldsymbol{u}}(\boldsymbol{u}(\boldsymbol{\xi}, T-t), \boldsymbol{\xi}, T-t) & & \left(\boldsymbol{\xi} \in S^{\mathrm{obs}}, 0 \leq t \leq T\right), \\
\boldsymbol{t}[\hat{\boldsymbol{u}}](\boldsymbol{\xi}, t) & =\mathbf{0} & & \left(\boldsymbol{\xi} \in S_{\mathrm{N}} \backslash S^{\text {obs }}, 0 \leq t \leq T\right), \\
\hat{\boldsymbol{u}}(\boldsymbol{\xi}, t) & =\mathbf{0} & & \left(\boldsymbol{\xi} \in S_{\mathrm{D}}, 0 \leq t \leq T\right), \\
\hat{\boldsymbol{u}}(\boldsymbol{\xi}, 0)=\dot{\hat{\boldsymbol{u}}}(\boldsymbol{\xi}, 0) & =\mathbf{0} & & (\boldsymbol{\xi} \in \Omega)
\end{aligned}
$$

On applying the reciprocity identity (15) over the punctured domain $V=\Omega_{\varepsilon}$ with boundary $\partial V=S \cup \Gamma_{\varepsilon}$, at the particular time instant $t=T$, and for the fields $\boldsymbol{w}_{a}=\hat{\boldsymbol{u}}$ and $\boldsymbol{w}_{b}=\tilde{\boldsymbol{u}}^{\varepsilon}$, one obtains the identity

$$
\begin{aligned}
\int_{0}^{T} \int_{S^{\mathrm{obs}}} \frac{\partial \varphi}{\partial \boldsymbol{u}}(\boldsymbol{u}(\boldsymbol{\xi}, \tau), \boldsymbol{\xi}, t) \cdot \tilde{\boldsymbol{u}}^{\varepsilon}(\boldsymbol{\xi}, \tau) \mathrm{d} \Gamma_{\xi} \mathrm{d} \tau \\
+\int_{\Gamma_{\varepsilon}}\left\{\boldsymbol{t}[\hat{\boldsymbol{u}}] \star \tilde{\boldsymbol{u}}^{\varepsilon}+\boldsymbol{t}[\boldsymbol{u}] \star \hat{\boldsymbol{u}}\right\}(\boldsymbol{\xi}, T) \mathrm{d} \Gamma_{\xi}=0
\end{aligned}
$$

where use has been made of the boundary conditions featured in the sets of governing equations (11) and (18), and of the fact that

$$
\begin{aligned}
\int_{S^{\mathrm{obs}}}\left\{\boldsymbol{t}[\hat{\boldsymbol{u}}] \star \tilde{\boldsymbol{u}}^{\varepsilon}\right\}(\boldsymbol{\xi}, T) \mathrm{d} \Gamma_{\xi}= & \int_{0}^{T} \int_{S^{\mathrm{obs}}} \boldsymbol{t}[\hat{\boldsymbol{u}}](\boldsymbol{\xi}, T-\tau) \cdot \tilde{\boldsymbol{u}}^{\varepsilon}(\boldsymbol{\xi}, \tau) \mathrm{d} \Gamma_{\xi} \mathrm{d} \tau \\
& =\int_{0}^{T} \int_{S^{\mathrm{obs}}} \frac{\partial \varphi}{\partial \boldsymbol{u}}(\boldsymbol{u}(\boldsymbol{\xi}, \tau), \boldsymbol{\xi}, t) \cdot \tilde{\boldsymbol{u}}^{\varepsilon}(\boldsymbol{\xi}, \tau) \mathrm{d} \Gamma_{\xi} \mathrm{d} \tau
\end{aligned}
$$

After substitution of (19), expansion (13) of $J\left(B_{\varepsilon}\right)$ hence takes the form

$$
J\left(\Omega_{\varepsilon}\right)=J(\Omega)-\int_{\Gamma_{\varepsilon}}\left\{\boldsymbol{t}[\hat{\boldsymbol{u}}] \star \tilde{\boldsymbol{u}}^{\varepsilon}+\boldsymbol{t}[\boldsymbol{u}] \star \hat{\boldsymbol{u}}\right\}(\boldsymbol{\xi}, T) \mathrm{d} \Gamma_{\xi}+o\left(\left|\tilde{\boldsymbol{u}}^{\varepsilon}\right|\right)
$$

in which the scattered field now appears only through its trace on the vanishing surface $\Gamma_{\varepsilon}$. 
Now, the leading contribution of each integral in expansion (20) as $\varepsilon \rightarrow 0$ needs to be evaluated. Noting that both the free-field and the adjoint field are defined on the uncavitated domain $\Omega$, the first integral in (20) can be recast as a volume integral over $B_{\varepsilon}$ via the divergence formula:

$$
\begin{aligned}
\int_{\Gamma_{\varepsilon}}\{\boldsymbol{t}[\boldsymbol{u}] \star \hat{\boldsymbol{u}}\}(\boldsymbol{\xi}, T) \mathrm{d} \Gamma_{\xi} & =-\int_{B_{\varepsilon}} \nabla \cdot\{(\mathcal{C}: \nabla \boldsymbol{u}) \star \hat{\boldsymbol{u}}\}(\boldsymbol{\xi}, T) \mathrm{d} V_{\xi} \\
& =-\int_{B_{\varepsilon}}\{\nabla \boldsymbol{u} \star(\mathcal{C}: \nabla \hat{\boldsymbol{u}})+\rho \dot{\boldsymbol{u}} \star \dot{\hat{\boldsymbol{u}}}\}(\boldsymbol{\xi}, T) \mathrm{d} V_{\xi}
\end{aligned}
$$

where the last equality makes use of the governing field equation (10a) for $\boldsymbol{u}$ and the property (17) of the time convolution. In the limit of an infinitesimal cavity, on therefore obtains

$$
\int_{\Gamma_{\varepsilon}}\{\boldsymbol{t}[\boldsymbol{u}] \star \hat{\boldsymbol{u}}\}(\boldsymbol{\xi}, T) \mathrm{d} \Gamma_{\xi}=-\varepsilon^{3}|\mathscr{B}|\{\nabla \boldsymbol{u} \star(\mathcal{C}: \nabla \hat{\boldsymbol{u}})+\rho \dot{\boldsymbol{u}} \star \dot{\hat{\boldsymbol{u}}}\}\left(\boldsymbol{x}^{\circ}, T\right)+o\left(\varepsilon^{3}\right)
$$

To evaluate the leading contribution of the second integral in expansion (20), it is necessary to determine the asymptotic behaviour on $\Gamma_{\varepsilon}$ of $\tilde{\boldsymbol{u}}^{\varepsilon}$ in the limit $\varepsilon \rightarrow 0$.

\subsection{Leading contribution of $\tilde{\boldsymbol{u}}^{\varepsilon}$ on $\Gamma_{\varepsilon}$ as $\varepsilon \rightarrow 0$}

To address this issue, it is convenient to reformulate the governing boundaryinitial problem (11) in terms of a boundary integral equation (BIE). The standard displacement BIE formulation for problem (11), expressed here for convenience as a pair of integral equations, reads [19-21]

$$
\begin{aligned}
& \frac{1}{2} \tilde{u}_{k}^{\varepsilon}(\boldsymbol{x}, t)+\mathrm{P} . \mathrm{V} . \int_{S_{\mathrm{N}}} \boldsymbol{T}^{k}(\boldsymbol{x}, t, \boldsymbol{\xi} ; \boldsymbol{n}) \star \tilde{\boldsymbol{u}}^{\varepsilon}(\boldsymbol{\xi}, t) \mathrm{d} \Gamma_{\xi} \\
& \quad-\int_{S_{\mathrm{D}}} \boldsymbol{U}^{k}(\boldsymbol{x}, t, \boldsymbol{\xi}) \star \boldsymbol{t}\left[\tilde{\boldsymbol{u}}^{\varepsilon}\right](\boldsymbol{\xi}, t) \mathrm{d} \Gamma_{\xi} \\
& \quad+\int_{\Gamma_{\varepsilon}} \boldsymbol{T}^{k}(\boldsymbol{x}, t, \boldsymbol{\xi} ; \boldsymbol{n}) \star \tilde{\boldsymbol{u}}^{\varepsilon}(\boldsymbol{\xi}, t) \mathrm{d} \Gamma_{\xi} \\
& \quad+\int_{\Gamma_{\varepsilon}} \boldsymbol{U}^{k}(\boldsymbol{x}, t, \boldsymbol{\xi}) \star \boldsymbol{t}[\boldsymbol{u}](\boldsymbol{\xi}, t) \mathrm{d} \Gamma_{\xi}=0 \quad(\boldsymbol{x} \in S, t \geq 0) \\
& \frac{1}{2} \tilde{u}_{k}^{\varepsilon}(\boldsymbol{x}, t)+\mathrm{P} . \mathrm{V} \cdot \int_{\Gamma_{\varepsilon}} \boldsymbol{T}^{k}(\boldsymbol{x}, t, \boldsymbol{\xi} ; \boldsymbol{n}) \star \tilde{\boldsymbol{u}}^{\varepsilon}(\boldsymbol{\xi}, t) \mathrm{d} \Gamma_{\xi} \\
& \quad+\int_{\Gamma_{\varepsilon}} \boldsymbol{U}^{k}(\boldsymbol{x}, t, \boldsymbol{\xi}) \star \boldsymbol{t}[\boldsymbol{u}](\boldsymbol{\xi}, t) \mathrm{d} \Gamma_{\xi} \\
& \quad+\int_{S_{\mathrm{N}}} \boldsymbol{T}^{k}(\boldsymbol{x}, t, \boldsymbol{\xi} ; \boldsymbol{n}) \star \tilde{\boldsymbol{u}}^{\varepsilon}(\boldsymbol{\xi}, t) \mathrm{d} \Gamma_{\xi} \quad\left(\boldsymbol{x} \in \Gamma_{\varepsilon}, t \geq 0\right) \\
& \quad-\int_{S_{\mathrm{D}}} \boldsymbol{U}^{k}(\boldsymbol{x}, t, \boldsymbol{\xi}) \star \boldsymbol{t}\left[\tilde{\boldsymbol{u}}^{\varepsilon}\right](\boldsymbol{\xi}, t) \mathrm{d} \Gamma_{\xi}=0 \quad
\end{aligned}
$$

where $\boldsymbol{U}^{k}(\boldsymbol{x}, \tau, \boldsymbol{\xi})$ and $\boldsymbol{T}^{k}(\boldsymbol{x}, \tau, \boldsymbol{\xi} ; \boldsymbol{n})$ are the elastodynamic fundamental solution, i.e. the displacement and traction vectors at $\boldsymbol{\xi}$ and time $\tau$ due to a 
unit impulsive point force acting at $\boldsymbol{x}$ in the $k$ direction and at time $t=0$ in an unbounded elastic medium, and 'P.V.' indicates that the corresponding integral, which is strongly singular at $\boldsymbol{\xi}=\boldsymbol{x}$, is defined in the Cauchy principal value (CPV) sense. Numerical implementations based on either direct evaluation of the CPV integrals [22] or indirect regularization based on rigid-body identities $[23,24]$ are well documented and will not be discussed further here.

As was done in previous investigations for time-harmonic problems [10,11], equations governing the leading contribution of $\tilde{\boldsymbol{u}}^{\varepsilon}$ on $\Gamma_{\varepsilon}$ as $\varepsilon \rightarrow 0$ are sought as the asymptotic form of integral equations (22) and (23). For this purpose, scaled coordinates $\overline{\boldsymbol{x}}=\left(\boldsymbol{x}-\boldsymbol{x}^{\circ}\right) / \varepsilon$ and $\overline{\boldsymbol{\xi}}=\left(\boldsymbol{\xi}-\boldsymbol{x}^{\circ}\right) / \varepsilon($ where $\overline{\boldsymbol{x}}, \overline{\boldsymbol{\xi}} \in \mathscr{S})$ are introduced when $\boldsymbol{x} \in \Gamma_{\varepsilon}$ or $\boldsymbol{\xi} \in \Gamma_{\varepsilon}$, respectively. In particular, one has

$$
\mathrm{d} \Gamma_{\xi}=\varepsilon^{2} \mathrm{~d} \bar{\Gamma}_{\bar{\xi}} \quad\left(\boldsymbol{\xi} \in \Gamma_{\varepsilon}, \overline{\boldsymbol{\xi}} \in \mathscr{S}\right)
$$

where $\mathrm{d} \bar{\Gamma}_{\bar{\xi}}$ denotes a differential element of the normalized surface $\mathscr{S}$.

It is natural to assume tentatively that the traces of $\tilde{\boldsymbol{u}}^{\varepsilon}$ on $\Gamma_{\varepsilon}$ and $S$ have asymptotic forms

$$
\begin{aligned}
\tilde{\boldsymbol{u}}^{\varepsilon}(\boldsymbol{\xi}, t)=\varepsilon^{d_{\Gamma}} \boldsymbol{V}(\overline{\boldsymbol{\xi}}, t)+o\left(\varepsilon^{d_{\Gamma}}\right) & \left(\boldsymbol{\xi} \in \Gamma_{\varepsilon}\right) \\
\tilde{\boldsymbol{u}}^{\varepsilon}(\boldsymbol{\xi}, t)=\varepsilon^{d_{\mathrm{S}}} \boldsymbol{W}(\boldsymbol{\xi}, t)+o\left(\varepsilon^{d_{\mathrm{S}}}\right) & (\boldsymbol{\xi} \in S)
\end{aligned}
$$

where the leading orders $d_{\Gamma}$ and $d_{\mathrm{S}}$ of the expansions and the auxiliary fields $\boldsymbol{V}(\overline{\boldsymbol{\xi}}, t), \boldsymbol{W}(\boldsymbol{\xi}, t)$ are to be determined.

Upon substitution of (25) and using (24), integral equation (22) takes the form

$$
\varepsilon^{d_{\mathrm{S}}} \mathcal{L}_{S S}(\boldsymbol{W})+\varepsilon^{d_{\Gamma}+2} \mathcal{L}_{S \mathscr{S}}(\boldsymbol{V})=O\left(\varepsilon^{3}\right)
$$

where the estimate in the right-hand side stems from

$$
\begin{aligned}
& -\int_{\Gamma_{\varepsilon}} \boldsymbol{U}^{k}(\boldsymbol{x}, t, \boldsymbol{\xi}) \star \boldsymbol{t}[\boldsymbol{u}](\boldsymbol{\xi}, t) \mathrm{d} \Gamma_{\xi} \\
= & \varepsilon^{3}|\mathscr{B}|\left\{\rho \dot{\boldsymbol{u}}\left(\boldsymbol{x}^{\circ}, t\right) \star \dot{\boldsymbol{U}}^{k}\left(\boldsymbol{x}, t, \boldsymbol{x}^{\circ}\right)+\nabla_{\xi} \boldsymbol{U}^{k}\left(\boldsymbol{x}, t, \boldsymbol{x}^{\circ}\right) \star\left(\mathcal{C}: \nabla_{\xi} \boldsymbol{u}\left(\boldsymbol{x}^{\circ}, t\right)\right)\right\}+o\left(\varepsilon^{3}\right)
\end{aligned}
$$

which is established similarly to $(21)$.

The asymptotic form of integral equation (23) also stems from a substitution of (25). In addition, the scaled coordinates $\overline{\boldsymbol{x}}, \overline{\boldsymbol{\xi}}$ are introduced in the integrals over $\Gamma_{\varepsilon}$. At this point, a key remark is that the fundamental displacement and traction can be shown to have the asymptotic behaviour

$$
\begin{aligned}
\boldsymbol{U}^{k}(\boldsymbol{x}, t, \boldsymbol{\xi}) \star \boldsymbol{f}(\boldsymbol{\xi}, t) & =\frac{1}{\varepsilon} \boldsymbol{U}^{k}(\overline{\boldsymbol{x}}, \overline{\boldsymbol{\xi}}) \cdot \boldsymbol{f}\left(\boldsymbol{x}^{\circ}, t\right)+O(1) \\
\boldsymbol{T}^{k}(\boldsymbol{x}, t, \boldsymbol{\xi} ; \boldsymbol{n}) \star \boldsymbol{g}(\boldsymbol{\xi}, t) & =\frac{1}{\varepsilon^{2}} \boldsymbol{T}^{k}(\overline{\boldsymbol{x}}, \overline{\boldsymbol{\xi}} ; \boldsymbol{n}) \cdot \boldsymbol{g}\left(\boldsymbol{x}^{\circ}, t\right)+O(1)
\end{aligned}
$$


where $\boldsymbol{U}^{k}(\overline{\boldsymbol{x}}, \overline{\boldsymbol{\xi}})$ and $\boldsymbol{T}^{k}(\overline{\boldsymbol{x}}, \overline{\boldsymbol{\xi}} ; \boldsymbol{n})$ are, respectively, the displacement and traction associated with the elastostatic Kelvin fundamental solution. By virtue of this property, the asymptotic form of integral equation (23) is thus

$$
\begin{aligned}
\varepsilon^{d_{\Gamma}} & \left\{\frac{1}{2} V_{k}(\overline{\boldsymbol{x}}, t)+\mathrm{P} . \mathrm{V} \cdot \int_{\mathscr{S}} \boldsymbol{T}^{k}(\overline{\boldsymbol{x}}, \overline{\boldsymbol{\xi}} ; \boldsymbol{n}) \cdot \boldsymbol{V}(\overline{\boldsymbol{\xi}}, t) \mathrm{d} \bar{\Gamma}_{\bar{\xi}}\right\} \\
+ & \varepsilon^{d_{\mathrm{S}}}\left\{\int_{S_{\mathrm{N}}} \boldsymbol{T}^{k}\left(\boldsymbol{x}^{\circ}, t, \boldsymbol{\xi} ; \boldsymbol{n}\right) \star \boldsymbol{W}(\boldsymbol{\xi}, t) \mathrm{d} \Gamma_{\xi}-\int_{S_{\mathrm{D}}} \boldsymbol{U}^{k}\left(\boldsymbol{x}^{\circ}, t, \boldsymbol{\xi}\right) \star \boldsymbol{t}[\boldsymbol{W}](\boldsymbol{\xi}, t) \mathrm{d} \Gamma_{\xi}\right\} \\
& =\varepsilon \boldsymbol{\sigma}\left(\boldsymbol{x}^{\circ}, t\right): \int_{\mathscr{B}} \nabla_{\bar{\xi}} \boldsymbol{U}^{k}(\overline{\boldsymbol{x}}, \overline{\boldsymbol{\xi}}) \mathrm{d} \bar{V}_{\bar{\xi}}+o\left(\varepsilon^{\min \left(d_{\Gamma}, d_{\mathrm{S}}, 1\right)}\right) \quad(\overline{\boldsymbol{x}} \in \mathscr{S}, t \geq 0)
\end{aligned}
$$

(where $\boldsymbol{\sigma}\left(\boldsymbol{x}^{\circ}, t\right)=\mathcal{C}: \nabla_{\xi} \boldsymbol{u}\left(\boldsymbol{x}^{\circ}, t\right)$ is the stress tensor associated with the free field), i.e. has the form

$$
\varepsilon^{d_{\Gamma}} \mathcal{L}_{\mathscr{S} \mathscr{S}} \boldsymbol{V}+\varepsilon^{d_{\mathrm{S}}} \mathcal{L}_{\mathscr{S} S} \boldsymbol{W}=O(\varepsilon)+o\left(\varepsilon^{\min \left(d_{\Gamma}, d_{\mathrm{S}}, 1\right)}\right)
$$

where the linear operators $\mathcal{L}_{\mathscr{S} \mathscr{S}}$ and $\mathcal{L}_{\mathscr{S} S}$ do not depend on $\varepsilon$ and the estimate in the right-hand side stems from

$$
\int_{\Gamma_{\varepsilon}} \boldsymbol{U}^{k}(\boldsymbol{x}, t, \boldsymbol{\xi}) \star \boldsymbol{t}[\boldsymbol{u}](\boldsymbol{\xi}, t) \mathrm{d} \Gamma_{\xi}=-\varepsilon \boldsymbol{\sigma}\left(\boldsymbol{x}^{\circ}, t\right): \int_{\mathscr{B}} \nabla_{\bar{\xi}} \boldsymbol{U}^{k}(\overline{\boldsymbol{x}}, \overline{\boldsymbol{\xi}}) \mathrm{d} \bar{V}_{\bar{\xi}}+o(\varepsilon)
$$

Now, $d_{\Gamma}$ and $d_{\mathrm{S}}$ must be chosen so that nonzero solutions $\boldsymbol{V}(\overline{\boldsymbol{\xi}}, t)$ and $\boldsymbol{W}(\boldsymbol{\xi}, t)$ are permitted in equations (26) and (29). Equation (29) then requires that $\min \left(d_{\Gamma}, d_{\mathrm{S}}\right)=1$. Moreover, since equation (26) with $d_{\mathrm{S}}=1$ implies that $\boldsymbol{W}=\mathbf{0}$, one must have $d_{\Gamma}=1$. Then, the lowest possible value for $d_{\mathrm{S}}$ is $d_{\mathrm{S}}=3$.

Summing up this analysis, the asymptotic form of $\tilde{\boldsymbol{u}}^{\varepsilon}(\boldsymbol{\xi}, t)$ for $\boldsymbol{\xi} \in \Gamma_{\varepsilon}$ is found to have the form

$$
\tilde{\boldsymbol{u}}^{\varepsilon}(\boldsymbol{\xi}, t)=\varepsilon \boldsymbol{V}(\overline{\boldsymbol{\xi}}, t)+o(\varepsilon) \quad(\overline{\boldsymbol{\xi}} \in \mathscr{S})
$$

where the auxiliary field $\boldsymbol{V}(\overline{\boldsymbol{\xi}}, t)$ solves the leading contribution to equation (28), which is found to be

$$
\begin{aligned}
\frac{1}{2} V_{k}(\overline{\boldsymbol{x}}, t)+\mathrm{P} . \mathrm{V} \cdot \int_{\mathscr{S}} & \boldsymbol{T}^{k}(\overline{\boldsymbol{x}}, \overline{\boldsymbol{\xi}} ; \boldsymbol{n}) \cdot \boldsymbol{V}(\overline{\boldsymbol{\xi}}, t) \mathrm{d} \bar{\Gamma}_{\bar{\xi}} \\
& =\boldsymbol{\sigma}\left(\boldsymbol{x}^{\circ}, t\right): \int_{\mathscr{B}} \nabla_{\bar{\xi}} \boldsymbol{U}^{k}(\overline{\boldsymbol{x}}, \overline{\boldsymbol{\xi}}) \mathrm{d} \bar{V}_{\bar{\xi}} \quad(\overline{\boldsymbol{x}} \in \mathscr{S}, t \geq 0)
\end{aligned}
$$

In fact, integral equation (31) turns out on inspection to be associated with the exterior elastostatic problem for the normalized cavity bounded by $\mathscr{S}$ embedded in an unbounded medium:

$$
\begin{aligned}
\nabla_{\bar{\xi}} \cdot\left(\mathcal{C}: \nabla_{\bar{\xi}} \boldsymbol{V}\right) & =\mathbf{0} & & \left(\overline{\boldsymbol{\xi}} \in \mathbb{R}^{3} \backslash \overline{\mathscr{B}}, t \geq 0\right), \\
\left(\mathcal{C}: \nabla_{\bar{\xi}} \boldsymbol{V}\right) \cdot \boldsymbol{n} & =-\boldsymbol{\sigma}\left(\boldsymbol{x}^{\mathrm{o}}, t\right) \cdot \boldsymbol{n} & & (\overline{\boldsymbol{\xi}} \in \mathscr{S}, t \geq 0)
\end{aligned}
$$

where $\boldsymbol{n}$ is the normal on $\mathscr{S}$ outward to $\mathbb{R}^{3} \backslash \overline{\mathscr{B}}$. Moreover, since the prescribed boundary traction is defined in terms of the spatially constant stress tensor 
$\boldsymbol{\sigma}\left(\boldsymbol{x}^{\circ}, t\right)$, the solution to (32) can be conveniently recast as

$$
\boldsymbol{V}(\overline{\boldsymbol{\xi}}, t)=\sigma_{k \ell}\left(\boldsymbol{x}^{\circ}, t\right) \mathcal{V}^{k \ell}(\overline{\boldsymbol{\xi}}) \quad\left(\overline{\boldsymbol{\xi}} \in \mathbb{R}^{3} \backslash \overline{\mathscr{B}}, t \geq 0\right)
$$

in terms of the individual solutions $\mathcal{V}^{k \ell}(\overline{\boldsymbol{\xi}})=\mathcal{V}^{\ell k}(\overline{\boldsymbol{\xi}})$ to six canonical problems:

$$
\begin{array}{ll}
\nabla_{\bar{\xi}} \cdot\left(\mathcal{C}: \nabla_{\bar{\xi}} \mathcal{V}^{k \ell}\right)=\mathbf{0} & \left(\overline{\boldsymbol{\xi}} \in \mathbb{R}^{3} \backslash \overline{\mathscr{B}}\right), \\
\left(\mathcal{C}: \nabla_{\bar{\xi}} \mathcal{V}^{k \ell}\right) \cdot \boldsymbol{n}=-\frac{1}{2}\left(n_{k} \boldsymbol{e}_{\ell}+n_{\ell} \boldsymbol{e}_{k}\right) & (\overline{\boldsymbol{\xi}} \in \mathscr{S})
\end{array}
$$

which are independent of $\boldsymbol{x}^{\circ}, \varepsilon$ and time.

\subsection{Topological derivative by the adjoint approach}

It is now possible to complete the evaluation of the leading contribution to the cost function expansion, equation (20). Using the results of Section 4.3, and in particular employing (24), (30) and (33) one has

$$
\begin{aligned}
\int_{\Gamma_{\varepsilon}}\{\boldsymbol{t} & {\left.[\hat{\boldsymbol{u}}] \star \tilde{\boldsymbol{u}}^{\varepsilon}\right\}(\boldsymbol{\xi}, T) \mathrm{d} \Gamma_{\xi} } \\
= & \varepsilon^{3} \int_{0}^{T} \int_{\mathscr{S}} \boldsymbol{n}(\overline{\boldsymbol{\xi}}) \cdot \hat{\boldsymbol{\sigma}}\left(\boldsymbol{x}^{\circ}+\varepsilon \boldsymbol{\xi}, \tau\right) \cdot \boldsymbol{V}(\overline{\boldsymbol{\xi}}, T-\tau) \mathrm{d} \bar{\Gamma}_{\bar{\xi}} \mathrm{d} \tau+o\left(\varepsilon^{3}\right) \\
= & \varepsilon^{3} \int_{0}^{T} \hat{\sigma}_{i j}\left(\boldsymbol{x}^{\mathrm{o}}, \tau\right) \sigma_{k \ell}\left(\boldsymbol{x}^{\mathrm{o}}, T-\tau\right)\left\{\int_{\mathscr{S}} n_{j}(\overline{\boldsymbol{\xi}}) \mathcal{V}_{i}^{k \ell}(\overline{\boldsymbol{\xi}}) \mathrm{d} \bar{\Gamma}_{\bar{\xi}}\right\}+o\left(\varepsilon^{3}\right)
\end{aligned}
$$

where $\hat{\boldsymbol{\sigma}}=\mathcal{C}: \nabla \hat{\boldsymbol{u}}$ is the stress tensor associated with the adjoint field.

Now, collecting partial results (21) and (35), one finally obtains the sought leading contribution to the expansion (20) of the objective function as a small cavity of radius $\varepsilon$ nucleates at a given location $\boldsymbol{x}^{\circ}$ :

$$
J\left(\Omega_{\varepsilon}\right)=J(\Omega)+\varepsilon^{3}|\mathscr{B}|\{\hat{\boldsymbol{\sigma}} \star(\mathcal{A}: \boldsymbol{\sigma})+\rho \dot{\boldsymbol{u}} \star \dot{\hat{\boldsymbol{u}}}\}\left(\boldsymbol{x}^{\circ}, T\right)+o\left(\varepsilon^{3}\right)
$$

where the constant fourth-order tensor $\mathcal{A}$ is defined by

$$
\mathcal{A}_{i j k \ell}=\frac{1}{2 \mu}\left[\frac{1-2 \nu}{1+\nu} \mathcal{J}_{i j k \ell}+\mathcal{K}_{i j k \ell}\right]-\frac{1}{|\mathscr{B}|} \int_{\mathscr{S}} n_{j}(\overline{\boldsymbol{\xi}}) \mathcal{V}_{i}^{k \ell}(\overline{\boldsymbol{\xi}}) \mathrm{d} \bar{\Gamma}_{\bar{\xi}}
$$

The only parameters affecting the solutions $\mathcal{V}^{k \ell}(\overline{\boldsymbol{\xi}})$ are the shape of $\mathscr{B}$ and the elastic tensor $\mathcal{C}$ of the reference medium. Hence, definition (37) implies that the tensor $\mathcal{A}$ depends only on $\mathscr{B}$ and $\mathcal{C}: \mathcal{A}=\mathcal{A}(\mathscr{B}, \mathcal{C})$.

On comparing expansion (36) to the definition (8) of the topological derivative $\mathcal{T}\left(\boldsymbol{x}^{\circ}\right)$, one finds that $\mathcal{T}\left(\boldsymbol{x}^{\circ}\right)$ and the function $\eta(\varepsilon)$ characterizing the asymp- 
totic behaviour of $J\left(\Omega_{\varepsilon}\right)-J(\Omega)$ are

$$
\begin{aligned}
\mathcal{T}\left(\boldsymbol{x}^{\circ}\right) & =\{\hat{\boldsymbol{\sigma}} \star(\mathcal{A}: \boldsymbol{\sigma})+\rho \dot{\boldsymbol{u}} \star \dot{\hat{\boldsymbol{u}}}\}\left(\boldsymbol{x}^{\circ}, T\right) \\
\eta(\varepsilon) & =\varepsilon^{3}|\mathscr{B}|
\end{aligned}
$$

It should be noted that the tensor $\mathcal{A}$ and the associated canonical problems (34) are the same as in the previous studies $[10,11]$ dealing with frequencydomain elastodynamics. For an arbitrarily shaped infinitesimal cavity, these canonical problems should in general be solved numerically. This is a modest computational task, since in fact one only needs to solve six elementary static exterior problems which do not depend on $\boldsymbol{x}^{\circ}$, for which the BEM is well suited. For the particular case of a spherical infinitesimal cavity (for which $\mathscr{B}$ is the unit ball, $\mathscr{S}$ is the unit sphere and $|\mathscr{B}|=4 \pi / 3$ ), problems (34) have an analytical solution, from which the following closed-form expression of $\mathcal{A}$ is obtained [10]:

$$
\mathcal{A}=\frac{1}{2 \mu}\left[\frac{3(1-\nu)}{2(1+\nu)} \mathcal{J}+\frac{15(1-\nu)}{7-5 \nu} \mathcal{K}\right]
$$

An alternative, equivalent, formulation for $\mathcal{T}\left(\boldsymbol{x}^{\circ}\right)$ involving the strains $\boldsymbol{\varepsilon}$ and $\hat{\varepsilon}$, rather than the stresses, associated with the free and adjoint fields is

$$
\mathcal{T}\left(\boldsymbol{x}^{\circ}\right)=\{\hat{\boldsymbol{\varepsilon}} \star(\mathcal{D}: \boldsymbol{\varepsilon})+\rho \dot{\boldsymbol{u}} \star \dot{\hat{\boldsymbol{u}}}\}\left(\boldsymbol{x}^{\circ}, T\right)
$$

where the fourth-order tensor $\mathcal{D}$ is given by

$$
\mathcal{D}=\mathcal{C}: \mathcal{A}: \mathcal{C}
$$

in the general case. In the particular case of a spherical infinitesimal cavity, this definition is easily evaluated with the help of (3), (4) and (5), to obtain the closed-form expression

$$
\mathcal{D}=2 \mu\left[\frac{3\left(1-\nu^{2}\right)}{2(1-2 \nu)^{2}} \mathcal{J}+\frac{15(1-\nu)}{7-5 \nu} \mathcal{K}\right]
$$

As explained in the previous study [11], in the case of ellipsoidal vanishing cavities, the tensor $\mathcal{A}$ can be expressed in terms of the Eshelby tensor associated with a uniform initial strain prescribed over an ellipsoidal region of same shape, providing exact expressions for $\mathcal{A}$ in all such cases. Moreover, on considering the limiting cases of infinitely thin ellipsoidal cavities, one should be able to establish topological sensitivity formulae for circular or elliptical infinitesimal cracks. 


\section{$5 \quad$ Formulation for scalar wave problems}

The topological derivative of an objective function can be developed along the same general lines as in Sections 3 and 4 for situations where the physics is described by the scalar wave equation (e.g. inversion of acoustic measurements). The main steps for this somewhat simpler setting are now summarized, in the context of linear acoustics for definiteness.

The reference domain $\Omega$ is now filled by a homogeneous medium characterized by the wave velocity $c$. The scattering of acoustic waves by rigid obstacles occupying a region $B$ bounded by $\Gamma$ is considered. The generic acoustic scattering problem is thus defined by the following set of equations:

$$
\mathcal{P}(\Gamma): \quad\left\{\begin{aligned}
{\left[\mathcal{L}(c) u_{\Gamma}\right](\boldsymbol{\xi}, t) } & =0 & & (\boldsymbol{\xi} \in \Omega, t \geq 0), \\
\nabla u_{\Gamma}(\boldsymbol{\xi}, t) \cdot \boldsymbol{n}(\boldsymbol{\xi}) & =p(\boldsymbol{\xi}, t) & & \left(\boldsymbol{\xi} \in S_{\mathrm{N}}, t \geq 0\right), \\
\nabla u_{\Gamma}(\boldsymbol{\xi}, t) \cdot \boldsymbol{n}(\boldsymbol{\xi}) & =0 & & (\boldsymbol{\xi} \in \Gamma, t \geq 0), \\
u_{\Gamma}(\boldsymbol{\xi}, t) & =0 & & \left(\boldsymbol{\xi} \in S_{\mathrm{D}}, t \geq 0\right), \\
u_{\Gamma}(\boldsymbol{\xi}, 0)=\dot{u}_{\Gamma}(\boldsymbol{\xi}, 0) & =0 & & (\boldsymbol{\xi} \in \Omega)
\end{aligned}\right.
$$

where $u(\boldsymbol{\xi}, t)$ is the acoustic pressure field, the given excitation $p(\boldsymbol{\xi}, t)$ is proportional to the normal wall acceleration, and $\mathcal{L}(c)$ is the governing partial differential operator of linear acoustics, i.e.

$$
[\mathcal{L}(c) w](\boldsymbol{\xi}, t)=\Delta w(\boldsymbol{\xi}, t)-\frac{1}{c^{2}} \ddot{w}(\boldsymbol{\xi}, t)
$$

Objective functions of format (6) are again considered, in terms of densities $\varphi\left(u_{\Gamma}(\boldsymbol{\xi}, t), \boldsymbol{\xi}, t\right)$ now dependent on the scalar acoustic pressure field $u_{\Gamma}(\boldsymbol{\xi}, t)$. With notations directly transposed from those of Sections 3 and 4, the topological derivative $\mathcal{T}\left(\boldsymbol{x}^{\circ}\right)$ is now to be identified on the basis of

$$
\int_{0}^{T} \int_{S^{\mathrm{obs}}} \frac{\partial \varphi}{\partial u}(u(\boldsymbol{\xi}, t), \boldsymbol{\xi}, t) \tilde{u}^{\varepsilon}(\boldsymbol{\xi}, t) \mathrm{d} \Gamma_{\xi} \mathrm{d} t=\mathcal{T}\left(\boldsymbol{x}^{\circ}\right) \eta(\varepsilon)+o(\eta(\varepsilon)) .
$$

Upon defining the adjoint solution $\hat{u}$ through the initial-boundary value problem

$$
\begin{aligned}
{[\mathcal{L}(c) \hat{u}](\boldsymbol{\xi}, t) } & =0 & & (\boldsymbol{\xi} \in \Omega, 0 \leq t \leq T), \\
\hat{u}_{, n}(\boldsymbol{\xi}, t) & =\frac{\partial \varphi}{\partial u}(u(\boldsymbol{\xi}, T-t), \boldsymbol{\xi}, T-t) & & \left(\boldsymbol{\xi} \in S^{\mathrm{obs}}, 0 \leq t \leq T\right), \\
\hat{u}_{, n}(\boldsymbol{\xi}, t) & =0 & & \left(\boldsymbol{\xi} \in S_{\mathrm{N}} \backslash S^{\mathrm{obs}}, 0 \leq t \leq T\right), \\
\hat{u}(\boldsymbol{\xi}, t) & =0 & & \left(\boldsymbol{\xi} \in S_{\mathrm{D}}, 0 \leq t \leq T\right), \\
\hat{u}(\boldsymbol{\xi}, 0)=\hat{\dot{u}}(\boldsymbol{\xi}, 0) & =0 & & (\boldsymbol{\xi} \in \Omega)
\end{aligned}
$$


(where $w_{, n}=\nabla w \cdot \boldsymbol{n}$ is the normal derivative of $w$ ) and using the reciprocity identity

$$
\int_{\partial V}\left\{w_{a, n} \star w_{b}-w_{b, n} \star w_{a}\right\}(\boldsymbol{\xi}, t) \mathrm{d} \Gamma_{\xi}=0 \quad(t \geq 0)
$$

instead of (15) for the states $\tilde{u}^{\varepsilon}$ and $\hat{u}$ in the same fashion than in Section 4, one obtains

$$
\begin{aligned}
\int_{0}^{T} & \int_{S^{\mathrm{obs}}} \frac{\partial \varphi}{\partial u}(u(\boldsymbol{\xi}, t), \boldsymbol{\xi}, t) \tilde{u}^{\varepsilon}(\boldsymbol{\xi}, t) \mathrm{d} \Gamma_{\xi} \mathrm{d} t \\
& =-\int_{\Gamma_{\varepsilon}}\left\{u u_{, n} \star \hat{u}+\hat{u}_{, n} \star \tilde{u}^{\varepsilon}\right\}(\boldsymbol{\xi}, T) \mathrm{d} \Gamma_{\xi}+o\left(\left\|\tilde{u}^{\varepsilon}\right\|\right) \\
& =\varepsilon^{3}|\mathscr{B}|\left\{\nabla u \star \nabla \hat{u}+\frac{1}{c^{2}} \dot{u} \star \dot{\hat{u}}\right\}\left(\boldsymbol{x}^{\circ}, T\right)-\int_{\Gamma_{\varepsilon}}\left\{\hat{u}_{, n} \star \tilde{u}^{\varepsilon}\right\}(\boldsymbol{\xi}, T) \mathrm{d} \Gamma_{\xi}+o\left(\left\|\tilde{u}^{\varepsilon}\right\|\right)
\end{aligned}
$$

The asymptotic behaviour of $\tilde{u}^{\varepsilon}(\boldsymbol{\xi}, t)$ is then found by seeking the limiting form as $\varepsilon \rightarrow 0$ of the pair of integral equations

$$
\begin{aligned}
& \frac{1}{2} \tilde{u}_{k}^{\varepsilon}(\boldsymbol{x}, t)+\int_{S_{\mathrm{N}}} G_{, n}(\boldsymbol{x}, t, \boldsymbol{\xi}) \star \tilde{u}^{\varepsilon}(\boldsymbol{\xi}, t) \mathrm{d} \Gamma_{\xi}-\int_{S_{\mathrm{D}}} G(\boldsymbol{x}, t, \boldsymbol{\xi}) \star \tilde{u}_{, n}^{\varepsilon}(\boldsymbol{\xi}, t) \mathrm{d} \Gamma_{\xi} \\
& \quad+\int_{\Gamma_{\varepsilon}} G_{, n}(\boldsymbol{x}, t, \boldsymbol{\xi}) \star \tilde{u}^{\varepsilon}(\boldsymbol{\xi}, t) \mathrm{d} \Gamma_{\xi} \\
& \quad+\int_{\Gamma_{\varepsilon}} G(\boldsymbol{x}, t, \boldsymbol{\xi}) \star \tilde{u}_{, n}^{\varepsilon}(\boldsymbol{\xi}, t) \mathrm{d} \Gamma_{\xi}=0 \quad(\boldsymbol{x} \in S, t \geq 0) \\
& \frac{1}{2} \tilde{u}_{k}^{\varepsilon}(\boldsymbol{x}, t)+\int_{\Gamma_{\varepsilon}} G_{, n}(\boldsymbol{x}, t, \boldsymbol{\xi}) \star \tilde{u}^{\varepsilon}(\boldsymbol{\xi}, t) \mathrm{d} \Gamma_{\xi}+\int_{\Gamma_{\varepsilon}} G(\boldsymbol{x}, t, \boldsymbol{\xi}) \star u_{, n}(\boldsymbol{\xi}, t) \mathrm{d} \Gamma_{\xi} \\
& \quad+\int_{S_{\mathrm{N}}} G, n(\boldsymbol{x}, t, \boldsymbol{\xi}) \star \tilde{u}^{\varepsilon}(\boldsymbol{\xi}, t) \mathrm{d} \Gamma_{\xi} \\
& \quad-\int_{S_{\mathrm{D}}} G(\boldsymbol{x}, t, \boldsymbol{\xi}) \star \tilde{u}_{, n}^{\varepsilon}(\boldsymbol{\xi}, t) \mathrm{d} \Gamma_{\xi}=0 \quad\left(\boldsymbol{x} \in \Gamma_{\varepsilon}, t \geq 0\right)
\end{aligned}
$$

where $G(\boldsymbol{x}, \tau, \boldsymbol{\xi})$ and $G_{, n}(\boldsymbol{x}, \tau, \boldsymbol{\xi})=\nabla_{\xi} G(\boldsymbol{x}, \tau, \boldsymbol{\xi}) \cdot \boldsymbol{n}(\boldsymbol{\xi})$ are the time-impulsive fundamental solution of the transient wave equation and its normal derivative. The latter have the asymptotic behaviour

$$
\begin{aligned}
G(\boldsymbol{x}, t, \boldsymbol{\xi}) \star f(\boldsymbol{\xi}, t) & =\frac{1}{\varepsilon} G(\overline{\boldsymbol{x}}, \overline{\boldsymbol{\xi}}) f\left(\boldsymbol{x}^{\circ}, t\right)+O(1) \\
G_{, n}(\boldsymbol{x}, t, \boldsymbol{\xi}) \star g(\boldsymbol{\xi}, t) & =\frac{1}{\varepsilon^{2}} G_{, n}(\overline{\boldsymbol{x}}, \overline{\boldsymbol{\xi}}) g\left(\boldsymbol{x}^{\circ}, t\right)+O(1)
\end{aligned}
$$

where $G(\overline{\boldsymbol{x}}, \overline{\boldsymbol{\xi}})$ is the fundamental solution of the Laplace equation. Note that, unlike in the elastodynamic case, all integrals in (48) and (49) are weakly singular at $\boldsymbol{\xi}=\boldsymbol{x}$.

The asymptotic form of $\tilde{u}^{\varepsilon}(\boldsymbol{\xi}, t)$ for $\boldsymbol{\xi} \in \Gamma_{\varepsilon}$ is found to have the form

$$
\tilde{u}^{\varepsilon}(\boldsymbol{\xi}, t)=\varepsilon V(\overline{\boldsymbol{\xi}}, t)+o(\varepsilon) \quad(\overline{\boldsymbol{\xi}} \in \mathscr{S})
$$


where the auxiliary field $V(\overline{\boldsymbol{\xi}}, t)$ is given by

$$
V(\overline{\boldsymbol{\xi}}, t)=u_{, k}\left(\boldsymbol{x}^{\mathrm{o}}, t\right) \mathcal{V}^{k}(\overline{\boldsymbol{\xi}})
$$

in terms of the individual solutions $\mathcal{V}^{k}(\overline{\boldsymbol{\xi}})$ to three canonical problems

$$
\Delta_{\bar{\xi}} \mathcal{V}^{k}=0 \quad\left(\overline{\boldsymbol{\xi}} \in \mathbb{R}^{3} \backslash \overline{\mathscr{B}}\right), \quad \nabla_{\bar{\xi}} \mathcal{V}^{k} \cdot \boldsymbol{n}=-n_{k} \quad(\overline{\boldsymbol{\xi}} \in \mathscr{S})
$$

which are independent of $\boldsymbol{x}^{\circ}, \varepsilon$ and time.

Finally, the topological derivative is given by

$$
\mathcal{T}\left(\boldsymbol{x}^{\circ}\right)=\left\{\nabla u \star(\boldsymbol{A} \cdot \nabla \hat{u})+\frac{1}{c^{2}} \dot{u} \dot{\hat{u}}\right\}\left(\boldsymbol{x}^{\circ}, T\right)
$$

with the second-order tensor $\boldsymbol{A}=\boldsymbol{A}(\mathscr{B})$ defined by

$$
A_{i k}=\delta_{i k}-\frac{1}{|\mathscr{B}|} \int_{\mathscr{S}} n_{i}(\overline{\boldsymbol{\xi}}) \mathcal{V}^{k}(\overline{\boldsymbol{\xi}}) \mathrm{d} \bar{\Gamma}_{\bar{\xi}}
$$

while, again, $\eta(\varepsilon)=\varepsilon^{3}|\mathscr{B}|$. For the particular case of infinitesimal objects of spherical shape (for which $\mathscr{B}$ is the unit ball, $\mathscr{S}$ the unit sphere and $|\mathscr{B}|=$ $4 \pi / 3)$, one finds

$$
V(\overline{\boldsymbol{\xi}})=-\frac{1}{2} \bar{\xi}_{k} \quad(\overline{\boldsymbol{\xi}} \in \mathscr{S}), \quad \boldsymbol{A}=\frac{3}{2} \boldsymbol{I}_{2}
$$

\section{Discussion}

\subsection{Choice of the reference body}

The notions of void-free reference configuration and the associated free field were postulated for clarity reasons but the applicability of topological sensitivity is not restricted to such geometric configurations. For example, the techniques developed in this study are equally applicable to reference solids containing pre-existing cavities and, in particular, to iterative imaging algorithms where the voids identified in a previous iteration are used to update the reference configuration for the next step.

\subsection{Formal similarities with parameter or shape sensitivity formulae}

The topological sensitivity formulae (38) and (52) are formally analogous to other formulae arising in parameter or shape sensitivity, which also feature 
an adjoint field and a time convolution. For instance, the formula expressing the perturbation $\delta J$ of a cost function of the form (6) induced by a given perturbation of the shape of the cavity boundary $\Gamma$ is [25]

$$
\delta J=\int_{\Gamma}\left[\boldsymbol{\sigma}_{\Gamma} \star \nabla \hat{\boldsymbol{u}}_{\Gamma}+\rho \dot{\boldsymbol{u}} \star \dot{\hat{\boldsymbol{u}}}_{\Gamma}\right](\boldsymbol{\xi}, T) \delta \Gamma(\boldsymbol{\xi}) \mathrm{d} \Gamma_{\xi}
$$

where the perturbed cavity shape is described through the mapping $\boldsymbol{\xi} \rightarrow \boldsymbol{\xi}+$ $\delta \Gamma(\boldsymbol{\xi}) \boldsymbol{n}(\boldsymbol{\xi})(\boldsymbol{\xi} \in \Gamma)$ and the forward and adjoint solutions $\boldsymbol{u}$ and $\hat{\boldsymbol{u}}_{\Gamma}$ are defined over the cavitated solid $\Omega_{\Gamma}$, through equations (1) and (18) supplemented with a traction-free boundary condition on $\Gamma$.

\subsection{Explicit expression of $\mathcal{T}\left(\boldsymbol{x}^{\circ}\right)$ in terms of Green's tensors}

Expression (38) is implicit in that it relies upon solutions of initial-boundary value problems on the reference body $\Omega$. Explicit expressions for $\mathcal{T}\left(\boldsymbol{x}^{\circ}\right)$ are obtained when the elastodynamic fundamental solution is a Green's tensor for $\Omega$, i.e. when it satisfies the boundary conditions

$$
\begin{aligned}
\boldsymbol{U}^{k}(\boldsymbol{\xi}, t, \boldsymbol{x})=\mathbf{0} & \left(\boldsymbol{\xi} \in S_{\mathrm{D}}, \boldsymbol{\xi} \neq \boldsymbol{x} ; \quad t \geq 0\right) \\
\boldsymbol{T}^{k}(\boldsymbol{\xi}, t, \boldsymbol{x} ; \boldsymbol{n})=\mathbf{0} & \left(\boldsymbol{\xi} \in S_{\mathrm{N}}, \boldsymbol{\xi} \neq \boldsymbol{x} ; \quad t \geq 0\right)
\end{aligned}
$$

In this case, the free and adjoint displacement fields are given by the explicit formulae

$$
\begin{aligned}
\boldsymbol{u}(\boldsymbol{x}, t) & =\int_{S_{\mathrm{N}}} \boldsymbol{U}^{k}(\boldsymbol{\xi}, t, \boldsymbol{x}) \star \boldsymbol{p}(\boldsymbol{\xi}, t) \mathrm{d} \Gamma_{\xi} \\
\hat{\boldsymbol{u}}(\boldsymbol{x}, t) & =\int_{S^{\mathrm{obs}}} \boldsymbol{U}^{k}(\boldsymbol{\xi}, t, \boldsymbol{x}) \star \frac{\partial \varphi}{\partial \boldsymbol{u}}(\boldsymbol{u}(\boldsymbol{\xi}, T-t), \boldsymbol{\xi}, T-t) \mathrm{d} \Gamma_{\xi}
\end{aligned}
$$

and the expression (38) becomes explicit as well.

Such Green's functions can be defined for bodies $\Omega$ of arbitrary shape, but are analytically known only in a few cases. Such cases include infinite media, for which formulae (54) still hold with a slightly different interpretation: the free field is now created by distributed sources over a surface $S_{\mathrm{N}}$ embedded in $\Omega=\mathbb{R}^{3}$, and the measurement surface $S^{\text {obs }}$ is also embedded in $\Omega$; besides, one does not need to have $S^{\text {obs }} \subseteq S_{\mathrm{N}}$ here.

Moreover, in cases where the free field does not satisfy radiation conditions at infinity (e.g. $u(\boldsymbol{\xi}, t)$ is a plane wave), representation (54) is no longer valid but the known free field can still be used in (38) together with the adjoint field given by (55). 


\subsection{Computational issues}

Although integral equation formulations have been useful in establishing formulae (38) or (52) for the topological derivative, they are by no means a mandatory tool for practical applications of these formulae. Any solution technique providing strain or stress distributions inside the medium can be invoked. In particular, using domain discretization methods such as the FEM or the FDM, the strain or stress distributions associated with the free and adjoint fields can be readily obtained. In the post-processing phase of FEM, recovery techniques may be used in order to enhance the accuracy of the computed field $\mathcal{T}\left(\boldsymbol{x}^{\circ}\right)$.

Using BEMs, the computation of the topological derivative is a two-step process requiring (i) the computation of the free and adjoint fields on the external boundary $S$, followed by (ii) the evaluation of integral reprsentation formulae at each search grid point $\boldsymbol{x}^{\circ}$. A very accurate evaluation of the field $\mathcal{T}\left(\boldsymbol{x}^{\circ}\right)$ can be expected. Both steps may entail high computational costs for fine search grids if traditional BEM techniques are utilized. Fast integral operator evaluation techniques such as the fast multipole method, which have been recently developed for time-domain scalar [26] and elastodynamic [27] wave problems, have the potential of bringing considerable computational savings for both steps (i) and (ii).

\section{$7 \quad$ Numerical example}

In this section, the application of the topological derivative to inverse problems based on the exploitation of measurements in the time domain is demonstrated on a numerical example in the context of 3D scalar wave propagation. A rigid spherical obstacle $B^{\text {true }}$ of radius $a$ is embedded in an unbounded acoustic medium with wave velocity $c$. The center of the obstacle is located at $\left(x_{1}^{\text {true }}, x_{2}^{\text {true }}, x_{3}^{\text {true }}\right)=(4,8,6) a$. The time histories of acoustic pressure are assumed to be recorded at point sensors located on the surface of a cube centered at the origin, whose faces lie in the planes $x_{i}= \pm 25 a(i=1,2,3)$ (i.e. with edges of length $50 a$ ). Three sensor configurations have been considered (hereinafter labelled $\left.S^{(1)}, S^{(2)}, S^{(3)}\right)$, where each face of the cube is divided into $1 \times 1,2 \times 2$ and $3 \times 3$ squares respectively and sensors are placed at all vertices of the squares thus defined. Hence, $S^{(1)}, S^{(2)}, S^{(3)}$ feature $N_{\mathrm{S}}=8,26,56$ sensors, respectively.

Three incident fields $u_{\mathrm{I}}(\boldsymbol{x}, t)(\mathrm{I}=1,2,3)$ have been considered, where

$$
u_{\mathrm{I}}(\boldsymbol{x}, t)=u_{0} \exp \left(-\frac{1}{2 a^{2}}\left(c t-x_{\mathrm{I}}\right)^{2}\right) \quad(\mathrm{I}=1,2,3)
$$


Thus, $u_{\mathrm{I}}(\boldsymbol{x}, t)$ is a plane wave travelling along the positive I-direction, of Gaussian shape, with a standard deviation equal to the radius of the sphere. Moreover, the time origin $t=0$ is such that the pulse has a maximum value at the center of $B^{\text {true }}$ at this instant. The synthetic data consist of the total pressures $\tilde{u}_{\mathrm{I}}^{\text {obs }}\left(\boldsymbol{x}^{m}, t\right)$ at the sensor locations $\boldsymbol{x}^{m}$ arising due to the interaction between the incident waves $u_{\mathrm{I}}(\boldsymbol{x}, t)$ and the actual obsctacle $B^{\text {true }}$, for each incident direction $I=1,2,3$. The objective function is then defined by

$$
J\left(\Omega_{\Gamma}\right)=\frac{1}{2} \sum_{\mathrm{I}=1}^{3} \sum_{m=1}^{N_{\mathrm{S}}} \int_{-\infty}^{+\infty}\left|\tilde{u}_{\mathrm{I}, \Gamma}\left(\boldsymbol{x}^{m}, t\right)-\tilde{u}_{\mathrm{I}}^{\mathrm{obs}}\left(\boldsymbol{x}^{m}, t\right)\right|^{2} \mathrm{~d} t=\sum_{\mathrm{I}=1}^{3} J_{\mathrm{I}}\left(\Omega_{\Gamma}\right)
$$

where $\tilde{u}_{\mathrm{I}, \Gamma}(\boldsymbol{x}, t)$ denotes the total field arising due to the incident wave $u_{\mathrm{I}}(\boldsymbol{x}, t)$ and an assumed obstacle of boundary $\Gamma$. Note that initial conditions are here rejected to the infinite past, hence the infinite bounds appearing in the time integral.

In the numerical results to follow, scattered fields at sensors have been evaluated by means of a semi-analytical technique based on separation of variables. Moreover, since the reference medium is unbounded and sensors are located on a discrete set of points, the adjoint field $\hat{u}_{\mathrm{I}}(\boldsymbol{\xi}, t)$ associated to each component $J_{\mathrm{I}}\left(\Omega_{\Gamma}\right)$ of $J\left(\Omega_{\Gamma}\right)$ is a superposition of fields created by appropriate point sources applied at the sensor locations:

$$
\hat{u}_{\mathrm{I}}(\boldsymbol{\xi}, t)=\sum_{m=1}^{N_{\mathrm{S}}} \frac{1}{4 \pi\left|\boldsymbol{\xi}-\boldsymbol{x}^{m}\right|}\left[\tilde{u}_{\mathrm{I}, \Gamma}-\tilde{u}_{\mathrm{I}}^{\mathrm{obs}}\right]\left(\boldsymbol{x}^{m}, T-t-\left|\boldsymbol{\xi}-\boldsymbol{x}^{m}\right| / c\right)
$$

having used the fact that

$$
G(\boldsymbol{x}, t, \boldsymbol{\xi})=\frac{\delta(t-|\boldsymbol{\xi}-\boldsymbol{x}| / c)}{4 \pi|\boldsymbol{\xi}-\boldsymbol{x}|}
$$

is the time-impulsive fundamental solution for the full space, with $\delta(t)$ denoting the 1D Dirac distribution.

Let $\mathcal{T}^{(\ell)}\left(\boldsymbol{x}^{\circ}\right)$ denote the topological derivative field associated with the sensor configuration $S^{(\ell)}$. The fields $\mathcal{T}^{(1)}\left(\boldsymbol{x}^{\circ}\right)$ and $\mathcal{T}^{(2)}\left(\boldsymbol{x}^{\circ}\right)$ have been computed on a regular search grid $\mathcal{G}$ made of $101 \times 101 \times 101$ points located on the vertices of a cubic grid bounded by the planes $x_{i}= \pm 20 a(i=1,2,3)$, so that the grid spacing along coordinate axes is $\Delta x^{\circ}=0.4 a$. The geometrical setup for this example is schematically depicted in figure 1.

Define $\mathcal{T}_{\text {min }}^{(\ell)}$ by

$$
\mathcal{T}_{\min }^{(\ell)}=\min _{\boldsymbol{x}^{\circ} \in \mathcal{G}} \mathcal{T}^{(\ell)}\left(\boldsymbol{x}^{\circ}\right)
$$

All computations performed led to $\mathcal{T}_{\text {min }}^{(\ell)}<0$, as expected. 
Figure 2 shows iso-surfaces of the field $\mathcal{T}^{(1)}\left(\boldsymbol{x}^{\circ}\right)$ computed on $\mathcal{G}$ corresponding to $\mathcal{T}^{(1)}=0.2 \mathcal{T}_{\text {min }}^{(1)}$ and $\mathcal{T}^{(1)}=0.6 \mathcal{T}_{\text {min }}^{(1)}$ (both values being negative). In both cases, values of $\mathcal{T}^{(1)}\left(\boldsymbol{x}^{\circ}\right)$ lower than the set isovalue are found to occur inside the iso-surfaces. The second iso-surface is found to enclose a region of space whose location agrees very well with that of the true obstacle, and of comparable size. This remark is emphasized by the iso-surface graphs of figure 3 , computed on a narrower and finer search grid $\mathcal{G}_{\text {closeup }}$ made of $41 \times 41 \times 41$ points located on the vertices of a cubic grid whose center coincides with that of the true obstacle and of half-width $2 a$, i.e. bounded by the planes $x_{i}=x_{i}^{\text {true }} \pm 2 a$ $(i=1,2,3)$. The grid spacing along coordinate axes is now $\Delta x^{\circ}=0.1 a$. Isosurfaces defined in the same way, obtained for the field $\mathcal{T}^{(2)}\left(\boldsymbol{x}^{\circ}\right)$, are shown in figure 4 for the wide grid $\mathcal{G}$ and figure 5 for the narrow grid $\mathcal{G}_{\text {closeup }}$. Results are similar, the main difference being in the improvement of the iso-surface corresponding to $\mathcal{T}^{(2)}=0.2 \mathcal{T}_{\text {min }}^{(2)}$ compared to that corresponding to $\mathcal{T}^{(1)}=0.2 \mathcal{T}_{\text {min }}^{(1)}$ due to the increased number of sensors. To emphasize the above remarks, figures 2 to 5 feature projections onto the three coordinate planes of the true scatterer (circles) and of the iso-surface (shaded zones).

Finally, figure 6 displays the fields $\mathcal{T}^{(\ell)}\left(\boldsymbol{x}^{\circ}\right)$ in the three planes parallel to the coordinate planes and passing through the center of $B^{\text {true }}$, for each sensor configuration $S^{(1)}, S^{(2)}, S^{(3)}$. Results are qualitatively similar, and emphasize the correct estimation of the location and size of $B^{\text {true }}$.

\section{Conclusions}

In this study, the concept of topological derivative, that has its origins in elastostatics and shape optimization, is extended to 3D elastic and acoustic-wave imaging of media of arbitrary geometry using data in the time domain. On taking the limiting form of the boundary integral equation governing the scattered field caused by a cavity with diminishing size, the topological derivative, which quantifies the sensitivity of the featured cost functional due to the creation of an infinitesimal hole (for the elastodynamic case) or rigid inclusion (for the acoustic case), is found to be expressed in terms of the time convolution of the free field and a supplementary adjoint field. This format, and in particular the time convolution element, is strongly reminiscent of formulae obtained elsewhere in connection with parameter or shape sensitivity analyses featuring time-dependent physical fields.

The proposed derivation of the topological derivative follows the generic pattern, already proposed in earlier investigations for 3D inverse scattering in the frequency domain, which is transposable to a variety of physical problems described by (i) other linear field equations such as heat transfer, and electromagnetics, or (ii) other types of infinitely small objects, such as inho- 
mogeneities or cracks.

A numerical example, featuring the analysis of synthetic data arising from the scattering of plane acoustic waves by a rigid spherical inclusion, shows that the spatial location of grid points where the topological derivative field reaches its lowest negative values (indicative of the tendency of the featured cost function to decrease, as desired in the context of an inverse problem, as the result of the nucleation of a small object at the corresponding search grid point) is consistent with the location of the true obstacle, and provides a reasonable estimation of the actual obstacle size. The information thus provided by analysis of the topological derivative field therefore appears to be useful in e.g. providing a good initial guess for performing a full-fledged, iterative, inversion procedure. If one accepts more qualitative results, topological derivative may also be defined for defining stand-alone imaging techniques, computationally faster than iterative inversion techniques.

\section{References}

[1] M. Bonnet, BIE and material differentiation applied to the formulation of obstacle inverse problems., Engng. Anal. with Bound. Elem. 15 (1995) 121136 .

[2] B. B. Guzina, S. Nintcheu Fata, M. Bonnet, On the stress-wave imaging of cavities in a semi-infinite solid, Int. J. Solids Struct. 40 (2003) 1505-1523.

[3] D. Colton, A. Kirsch, A simple method for solving inverse scattering problems in the resonance region, Inverse Problems 12 (1996) 383-393.

[4] S. Nintcheu Fata, B. B. Guzina, A linear sampling method for near-field inverse problems in elastodynamics, Inverse Problems 20 (2004) 713-736.

[5] H. A. Eschenauer, V. V. Kobelev, A. Schumacher, Bubble method for topology and shape optimization of structures, Structural Optimization 8 (1994) 42-51.

[6] A. Schumacher, Topologieoptimierung von Bauteilstrukturen unter Verwendung von Lochpositionierungskriterien, Ph.D. thesis, Univ. of Siegen, Germany (1995).

[7] M. P. Bendsoe, O. Sigmund, Topology optimization (second edition), SpringerVerlag, 2003.

[8] S. Garreau, P. Guillaume, M. Masmoudi, The topological asymptotic for PDE systems: the elasticity case., SIAM J. Contr. Opt. 39 (2001) 1756-1778.

[9] J. Sokolowski, A. Zochowski, On the topological derivative in shape optimization., SIAM J. Control Optim. 37 (1999) 1251-1272.

[10] B. B. Guzina, M. Bonnet, Topological derivative for the inverse scattering of elastic waves, Quart. J. Mech. Appl. Math. 57 (2004) 161-179. 
[11] M. Bonnet, B. B. Guzina, Sounding of finite solid bodies by way of topological derivative, Int. J. Num. Meth. in Eng. 61 (2004) 2344-2373.

[12] R. Gallego, G. Rus, Identification of cracks and cavities using the topological sensitivity boundary integral equation, in: Proc. IABEM 2002 Conference, Austin, Texas, CD-ROM, 2002.

[13] L. Jackowska-Strumillo, J. Sokolowski, A. Zochowski, Topological optimization and inverse problems, Computer Assisted Mechanics and Engineering Sciences 10 (2) (2002) 163-176.

[14] G. R. Feijóo, A new method in inverse scattering based on the topological derivative, Inverse Problems 20 (2004) 1819-1840.

[15] J. Haslinger, R. A. E. Mäkinen, Introduction to shape optimization: theory, approximation and computation, SIAM, Philadelphia, 2003.

[16] A. Tarantola, Inverse problem theory and methods for model parameter estimation, SIAM, 2005.

[17] Z. Michalewicz, D. B. Fogel, How to solve it: modern heuristics, Springer-Verlag, 2004.

[18] L. T. Wheeler, E. Sternberg, Some theorems in classical elastodynamics, Arch. Rat. Mech. Anal. 31 (1968) 51-90.

[19] A. C. Eringen, E. S. Suhubi, Elastodynamics (vol II - linear theory), Academic Press, 1975.

[20] S. Ahmad, P. K. Banerjee, Time-domain Transient elastodynamic analysis of 3-D solids by BEM., Int. J. Num. Meth. in Eng. 26 (1988) 1709-1728.

[21] D. E. Beskos, Boundary element methods in dynamic analysis, part. II (19861996)., Appl. Mech. Rev. 50 (1997) 149-197.

[22] M. Guiggiani, Formulation and numerical treatment of boundary integral equations with hypersingular kernels., in: V. Sladek, J. Sladek (Eds.), Singular Integrals in Boundary Element Methods, Comp. Mech. Publ., Southampton, 1998, Ch. 3, pp. 85-124.

[23] M. Tanaka, V. Sladek, J. Sladek, Regularization techniques applied to boundary element methods, Appl. Mech. Rev. 47 (1994) 457-499.

[24] M. Bonnet, Boundary Integral Equations Methods for Solids and Fluids, John Wiley and Sons, 1999.

[25] M. Bonnet, A general boundary-only formula for crack shape sensitivity of integral functionals., C.R. Acad. Sci. Paris, série II 327 (1999) 1215-1221.

[26] A. A. Ergin, B. Shanker, E. Michielssen, Fast evaluation of three-dimensional transient wave fields using diagonal translation operators, J. Comp. Phys. 146 (1998) 157-180.

[27] T. Takahashi, N. Nishimura, S. Kobayashi, A fast BIEM for three-dimensional elastodynamics in time domain, Engng. Anal. with Bound. Elem. 28 (2004) $165-180$. 


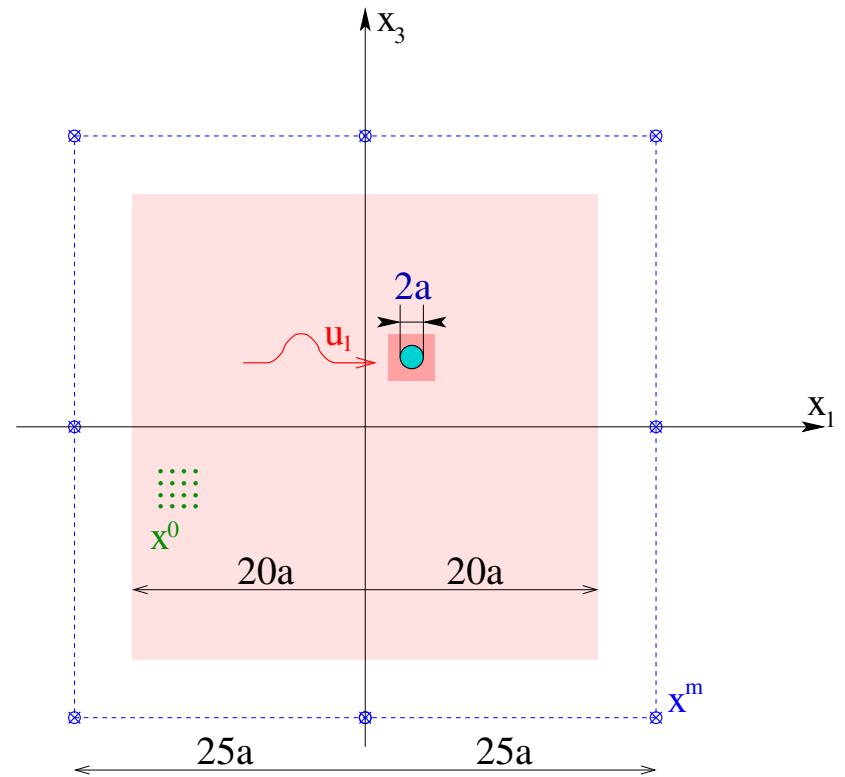

Fig. 1. Geometrical setup for the numerical example, viewed in the $\left(x_{1}, x_{3}\right)$-plane: rigid spherical obstacle, region occupied by the search grids $\mathcal{G}$ (shaded, light) and $\mathcal{G}^{\text {closeup }}$ (shaded), sensor locations (configuration $S^{(2)}$. 

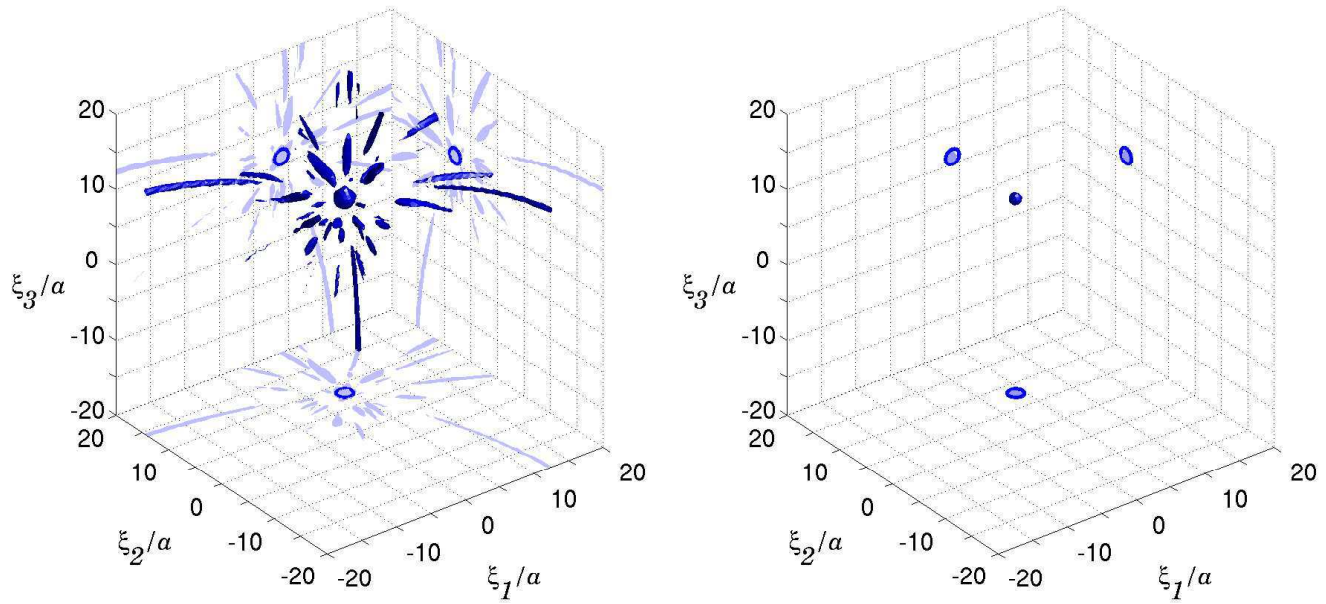

Fig. 2. Sensor configuration $S^{(1)}$ : iso-surfaces of $\mathcal{T}^{(1)}\left(\boldsymbol{x}^{\circ}\right)$ for $\mathcal{T}^{(1)}=0.2 \mathcal{T}_{\text {min }}^{(1)}$ (left) and $\mathcal{T}^{(1)}=0.6 \mathcal{T}_{\text {min }}^{(1)}$ (right), computed on the wide search grid $\mathcal{G}$ (values of $\mathcal{T}^{(1)}\left(\boldsymbol{x}^{\circ}\right)$ lower than the iso-value are inside the iso-surface). 

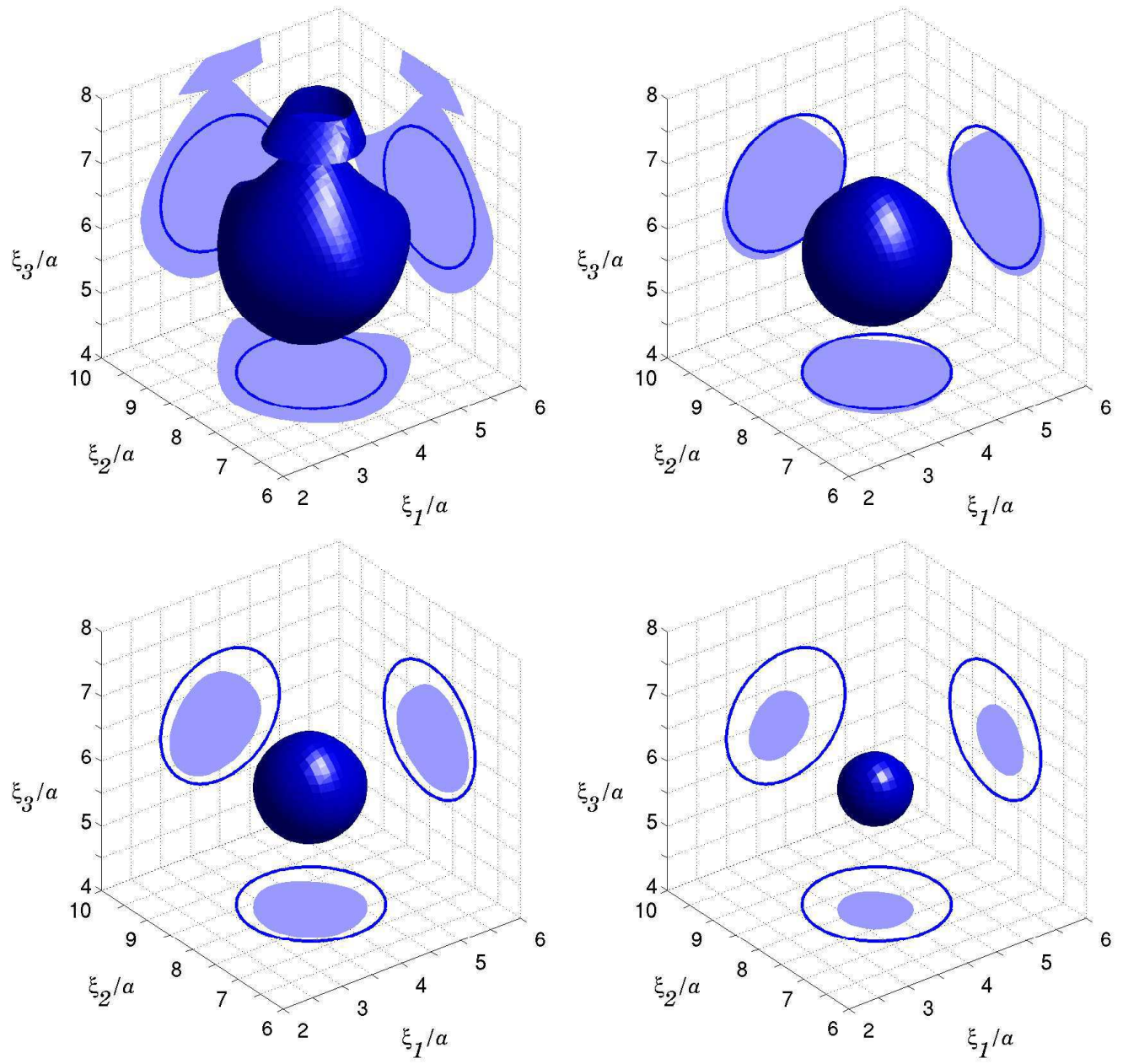

Fig. 3. Sensor configuration $S^{(1)}$ : iso-surfaces of $\mathcal{T}^{(1)}\left(\boldsymbol{x}^{\circ}\right)$ for $\mathcal{T}^{(1)}=0.2 \mathcal{T}_{\text {min }}^{(1)}$ (upper left), $\mathcal{T}^{(1)}=0.4 \mathcal{T}_{\text {min }}^{(1)}$ (upper right), $\mathcal{T}^{(1)}=0.6 \mathcal{T}_{\text {min }}^{(1)}$ (lower left) and $\mathcal{T}^{(1)}=0.8 \mathcal{T}_{\text {min }}^{(1)}$ (lower right), computed on the narrow search grid $\mathcal{G}^{\text {closeup }}$ (values of $\mathcal{T}^{(1)}\left(\boldsymbol{x}^{\circ}\right)$ lower than the iso-value are inside the iso-surface). 

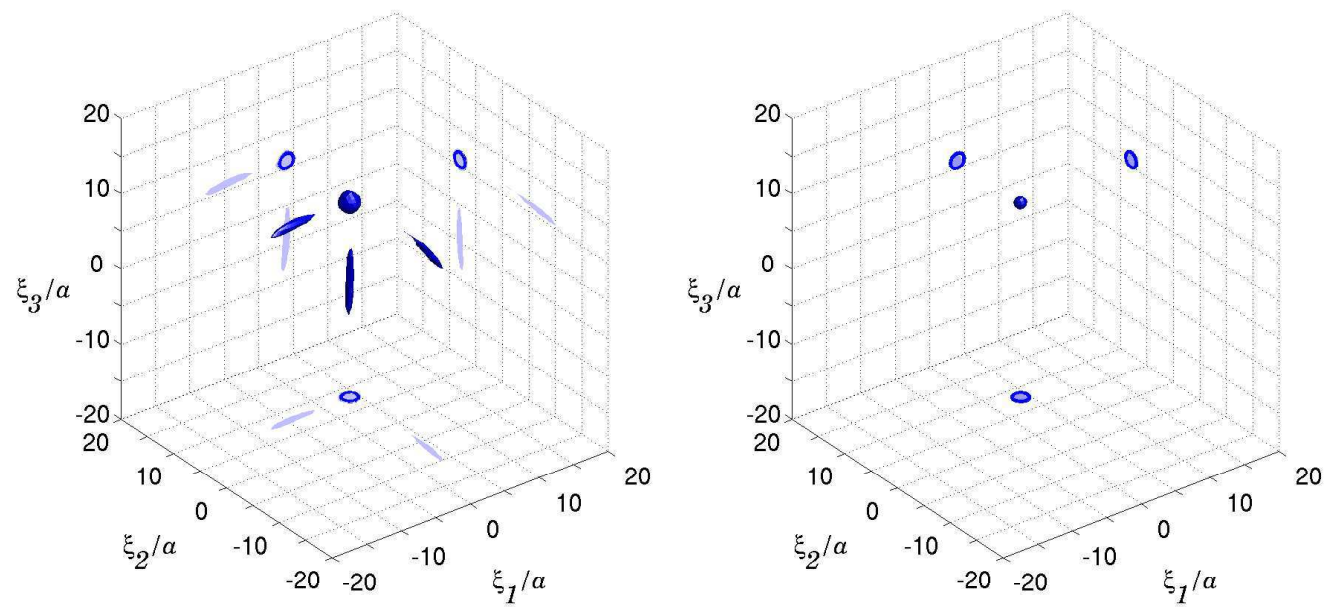

Fig. 4. Sensor configuration $S^{(2)}$ : iso-surfaces of $\mathcal{T}^{(2)}\left(\boldsymbol{x}^{\circ}\right)$ for $\mathcal{T}^{(2)}=0.2 \mathcal{T}_{\text {min }}^{(2)}$ (left) and $\mathcal{T}^{(2)}=0.6 \mathcal{T}_{\text {min }}^{(2)}$ (right), computed on the wide search grid $\mathcal{G}$ (values of $\mathcal{T}^{(2)}\left(\boldsymbol{x}^{\circ}\right)$ lower than the iso-value are inside the iso-surface). 

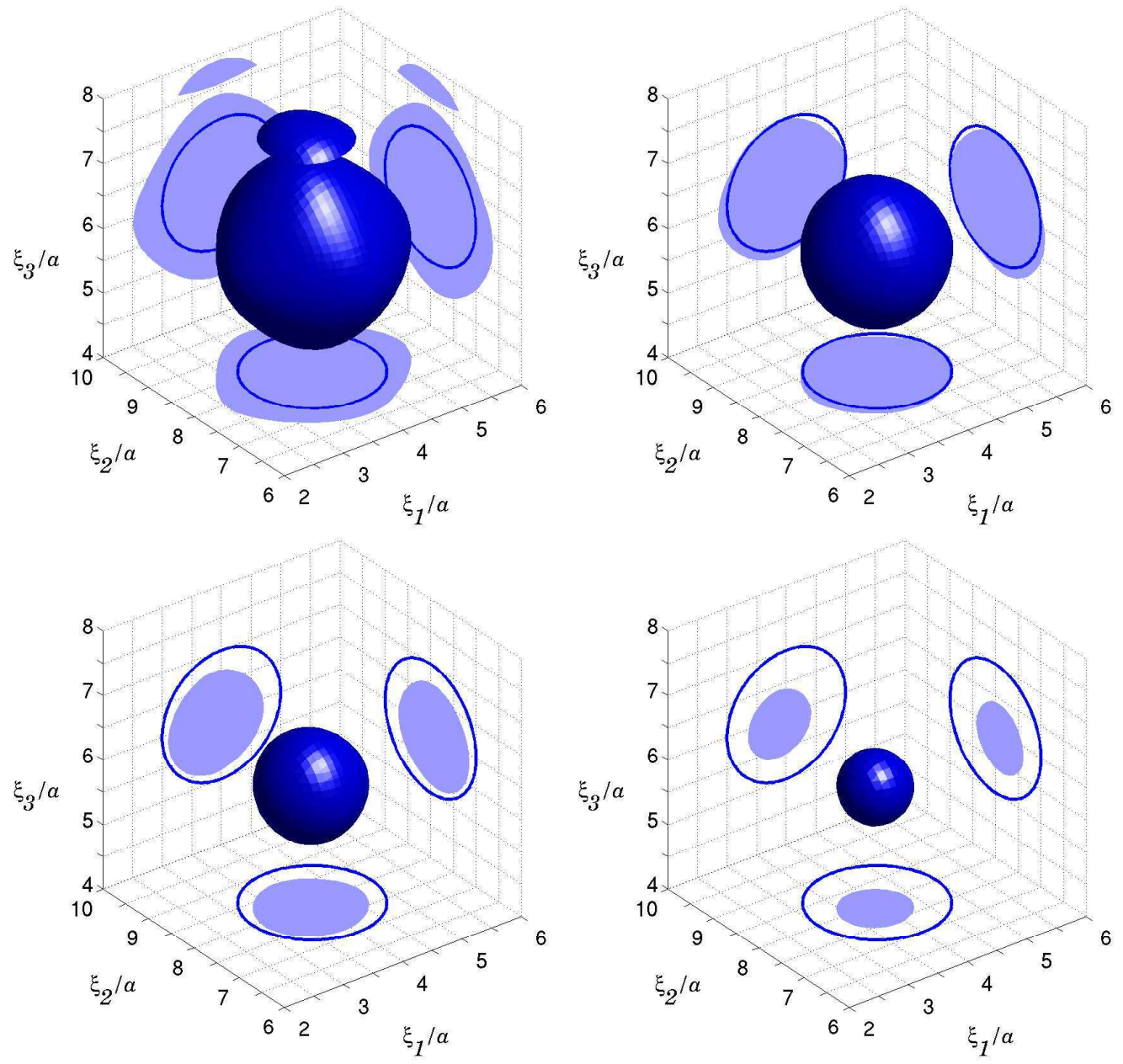

Fig. 5. Sensor configuration $S^{(2)}$ : iso-surfaces of $\mathcal{T}^{(2)}\left(\boldsymbol{x}^{\circ}\right)$ for $\mathcal{T}^{(2)}=0.2 \mathcal{T}_{\text {min }}^{(2)}$ (upper left), $\mathcal{T}^{(2)}=0.4 \mathcal{T}_{\text {min }}^{(2)}$ (upper right), $\mathcal{T}^{(2)}=0.6 \mathcal{T}_{\text {min }}^{(2)}$ (lower left) and $\mathcal{T}^{(2)}=0.8 \mathcal{T}_{\text {min }}^{(2)}$ (lower right), computed on the narrow search grid $\mathcal{G}^{\text {closeup }}$ (values of $\mathcal{T}^{(2)}\left(\boldsymbol{x}^{\circ}\right)$ lower than the iso-value are inside the iso-surface). 

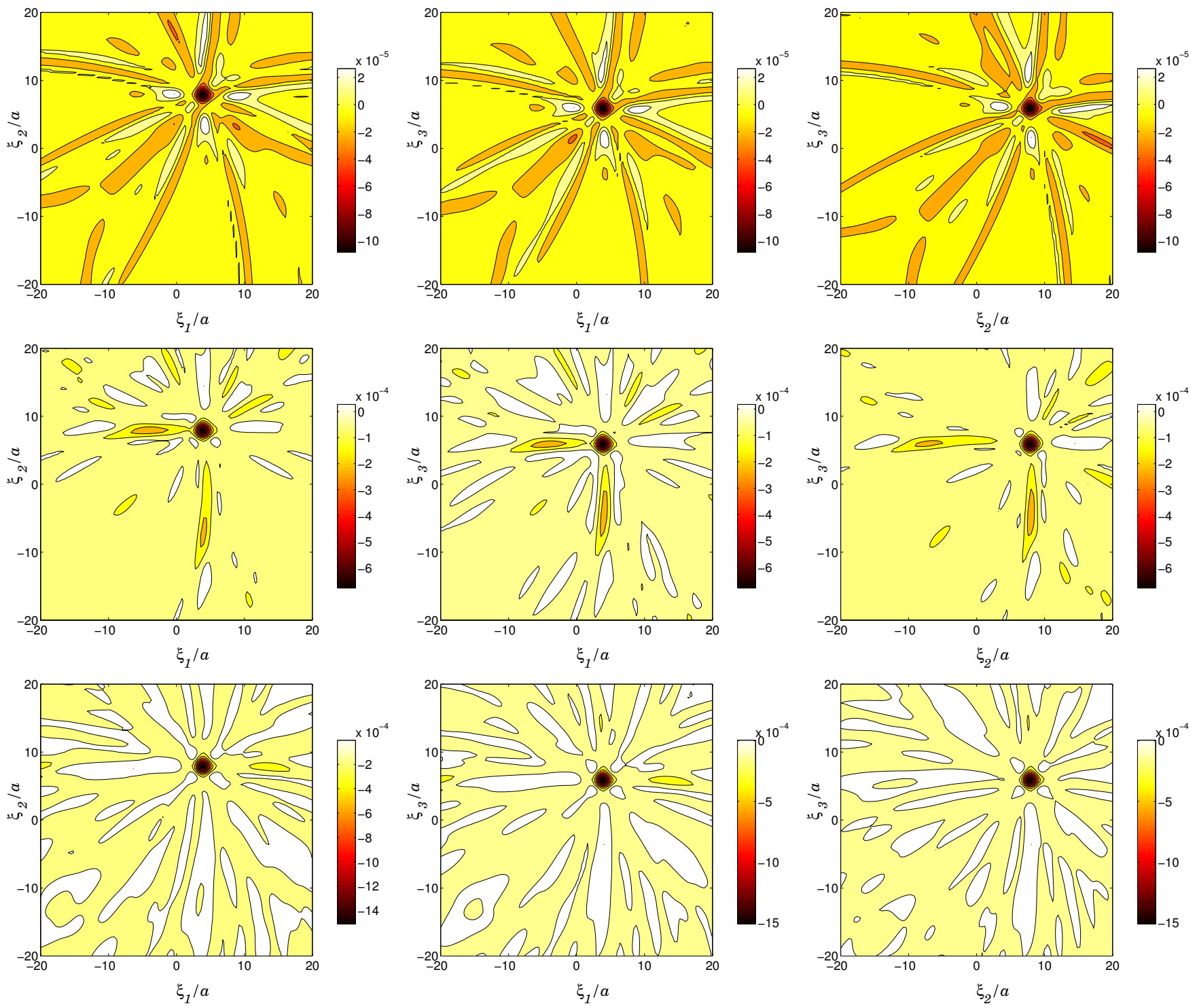

Fig. 6. Distribution of $\mathcal{T}^{(\ell)}\left(\boldsymbol{x}^{\circ}\right)$ in coordinate planes: $\ell=1$ (top row), $\ell=2$ (middle row) and $\ell=3$ (bottom row); planes $x_{3}=x_{3}^{\text {true }}$ (left column), $x_{2}=x_{2}^{\text {true }}$ (middle column) and $x_{1}=x_{1}^{\text {true }}$ (right column). 